$$
\begin{array}{lllll}
\lrcorner & \lrcorner
\end{array}
$$

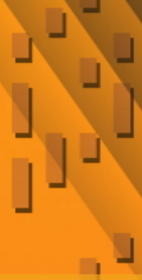

Equations for Chow and Hilbert quotients

Angela Gibney and Diane Maclagan 


\title{
Equations for Chow and Hilbert quotients
}

\author{
Angela Gibney and Diane Maclagan
}

\begin{abstract}
We give explicit equations for the Chow and Hilbert quotients of a projective scheme $X$ by the action of an algebraic torus $T$ in an auxiliary toric variety. As a consequence we provide geometric invariant theory descriptions of these canonical quotients, and obtain other GIT quotients of $X$ by variation of GIT quotient. We apply these results to find equations for the moduli space $\bar{M}_{0, n}$ of stable genus-zero $n$-pointed curves as a subvariety of a smooth toric variety defined via tropical methods.
\end{abstract}

\section{Introduction}

When a reductive group $G$ acts linearly on a projective scheme $X$, a fundamental problem is to describe a good notion of a quotient $X / G$. This question frequently arises in the construction and compactification of moduli spaces. In many situations, there is an open subset $U \subset X$ on which $G$ acts freely, such that a scheme $U / G$ exists as a geometric quotient. Constructing the quotient $X / G$ is thus choosing a good compactification of $U / G$. One way to compactify is by forming the Chow quotient $X / /{ }^{\mathrm{Ch}} G$ or Hilbert quotient $X / /{ }^{\mathrm{H}} G$ of $X$ by $G$ (see [Kapranov 1993]). These quotients are taken to be the closure of $U / G$ in an appropriate Chow variety or Hilbert scheme. They are natural canonical quotients with proper birational maps to any GIT (geometric invariant theory) quotient. See also [Hu 2005; Keel and Tevelev 2006].

In this paper we treat the case where $G=T^{d}$ is a $d$-dimensional algebraic torus acting equivariantly on a subscheme $X$ of $\mathbb{P}^{m}$. Given the ideal $I$ of $X$ as a subscheme of $\mathbb{P}^{m}$, we describe equations for $X / /^{\mathrm{Ch}} T^{d}$ and $X / /^{\mathrm{H}} T^{d}$ in the Cox rings of toric subvarieties of the Chow and Hilbert quotients of $\mathbb{P}^{m}$.

As a first application of our results, we give GIT constructions of $X /{ }^{\mathrm{Ch}} T^{d}$ and $X / /{ }^{\mathrm{H}} T^{d}$ and we prove that all GIT quotients of $X$ by $T^{d}$ can be obtained from the Chow and Hilbert quotients by variation of the GIT.

As a second application we study the action of an $(n-1)$-dimensional torus $T^{n-1}$ on the Grassmannian $G(2, n)$. Here we can take $U$ to be the points with

MSC2000: primary 14L30; secondary 14M25, 14L24, 14H10.

Keywords: Chow quotient, Hilbert quotient, moduli of curves, space of phylogenetic trees. 
nonvanishing Plücker coordinates, and the quotient $U / T^{n-1}$ is the moduli space $M_{0, n}$ of smooth $n$-pointed genus-zero curves. In this case the desired compactification is the celebrated moduli space $\bar{M}_{0, n}$ of stable $n$-pointed genus-zero curves. [Kapranov 1993] showed that $\bar{M}_{0, n}$ is isomorphic to both the Chow and Hilbert quotients of $G(2, n)$ by the $T^{n-1}$-action. We give explicit equations for $\bar{M}_{0, n}$ as a subvariety of a smooth toric variety $X_{\Delta}$ whose fan is the well-studied space of phylogenetic trees. We show that the equations for $\bar{M}_{0, n}$ in the Cox ring $S$ of $X_{\Delta}$ are generated by the Plücker relations homogenized with respect to the grading of $S$.

We now describe our results in more detail. The notation $X / /^{\star} T^{d}$ is used to refer to either the Chow or the Hilbert quotient. We assume that no irreducible component of $X$ lies in any coordinate subspace. This means that $X / /^{\star} T^{d}$ is a subscheme of $\mathbb{P}^{m} / /^{\star} T^{d}$. The quotient $\mathbb{P}^{m} / /^{\star} T^{d}$ is a not necessarily normal toric variety [Kapranov et al. 1992] whose normalization we denote by $X_{\Sigma^{\star}}$. By $X / \|_{n}^{\star} T^{d}$ we mean the pullback of $X / /^{\star} T^{d}$ to $X_{\Sigma^{\star}}$. Our main theorem, in slightly simplified form, is the following. This is proved in Theorems 3.2 and 4.6 and Proposition 4.3.

Theorem 1.1. Let $T^{d} \cong\left(\mathbb{k}^{\times}\right)^{d}$ act on $\mathbb{P}^{m}$ and let $X \subset \mathbb{P}^{m}$ be a $T^{d}$-equivariant subscheme with corresponding ideal $I(X)=\left\langle f_{1}, \ldots, f_{g}\right\rangle \subset \mathbb{k}\left[x_{0}, \ldots, x_{m}\right]$. Let $X_{\Sigma} \subset X_{\Sigma^{\star}}$ be any toric subvariety with $X \subseteq X_{\Sigma} \subseteq X_{\Sigma^{\star} \text {. }}$

(1) (Equations) The ideal I of the Hilbert or Chow quotient $X / /{ }_{n}^{\star} T^{d}$ in the Cox ring $S=\mathbb{k}\left[y_{1}, \ldots y_{r}\right]$ of $X_{\Sigma}$ can be computed effectively. Explicitly, $I$ is obtained by considering the $f_{i}$ as polynomials in $y_{1}, \ldots, y_{m+1}$, homogenizing them with respect to the $\mathrm{Cl}\left(X_{\Sigma}\right)$-grading of $S$, and then saturating the result by the product of all the variables in $S$.

(2) (GIT) There is a GIT construction of the Chow and Hilbert quotients of X, and these are related to the GIT quotients of $X$ by variation of the GIT quotient. This gives equations for all quotients in suitable projective embeddings. Let $H=\operatorname{Hom}\left(\mathrm{Cl}\left(X_{\Sigma}\right), \mathbb{k}^{\times}\right)$. There is a nonzero cone $\varphi \subset \mathrm{Cl}\left(X_{\Sigma}\right) \otimes \mathbb{R}$ for which $X / /{ }_{n}^{\star} T^{d}$ is the GIT quotient

$$
X / /_{n}^{\star} T^{d}=Z(I) / / \alpha H
$$

for any rational $\alpha \in \operatorname{relint}(G)$, where $Z(I)$ is the subscheme of $\mathbb{A}^{r}$ defined by I. For any GIT quotient $X / / \beta T^{d}$ of $X$, there are choices of $\alpha$ outside $\varphi$ for which $Z(I) / / \alpha H=X / / \beta T^{d}$.

A more precise formulation of the homogenization is given in Theorem 3.2 and Remark 3.3. We explain in Corollary 4.4 how each choice of $\alpha \in \operatorname{relint}(\mathscr{G})$ gives an embedding of $X / /_{n}^{\star} T^{d}$ into some projective space.

We use tropical algebraic geometry in the spirit of [Tevelev 2007] to embed $\bar{M}_{0, n}$ in a smooth toric variety $X_{\Delta}$. The combinatorial data describing $\Delta$ and the simple 
equations for $\bar{M}_{0, n}$ in the Cox ring of $X_{\Delta}$ are described in the following theorem. Let $[n]=\{1, \ldots, n\}$ and set $\mathscr{I}=\{I \subset[n]: 1 \in I,|I| \geq 2,|[n] \backslash I| \geq 2\}$. The set $\mathscr{I}$ indexes the boundary divisors of $\bar{M}_{0, n}$.

Theorem 1.2. Let $\Delta$ be the fan in $\mathbb{R}^{\left(\begin{array}{l}n \\ 2\end{array}\right)-n}$ described in Section 5 (the space of phylogenetic trees). The rays of $\Delta$ are indexed by the set $\Phi$.

(1) (Equations) Equations for $\bar{M}_{0, n}$ in the Cox ring $S=\mathbb{k}\left[x_{I}: I \in \mathscr{I}\right]$ of $X_{\Delta}$ are obtained by homogenizing the Plücker relations with respect to the grading of $S$ and then saturating by the product of the variables of $S$. Specifically, the ideal is

$$
I_{\bar{M}_{0, n}}=\left(\left\langle\prod_{\substack{i, j \in I \\ k, l \notin I}}-\prod_{\substack{i, k \in I \\ j, l \notin I}} x_{I}+\prod_{\substack{i, l \in I \\ j, k \notin I}} x_{I}\right\rangle:\left(\prod_{I} x_{I}\right)^{\infty}\right),
$$

where the generating set runs over all $\{i, j, k, l\}$ with $1 \leq i<j<k<l \leq n$, and $x_{I}=x_{[n] \backslash I}$ if $1 \notin I$.

(2) (GIT) There is a nonzero cone $\mathscr{G} \subset \mathrm{Cl}\left(X_{\Delta}\right) \otimes \mathbb{R} \cong \operatorname{Pic}\left(\bar{M}_{0, n}\right) \otimes \mathbb{R}$ for which for rational $\alpha \in \operatorname{int}(G)$ we have the GIT construction of $\bar{M}_{0, n}$ as

$$
\bar{M}_{0, n}=Z\left(I_{\bar{M}_{0, n}}\right) / / \alpha H,
$$

where $Z\left(I_{\bar{M}_{0, n}}\right) \subset \mathbb{A}^{|\mathscr{}|}$ is the affine subscheme defined by $I_{\bar{M}_{0, n}}$, and $H$ is the torus $\operatorname{Hom}\left(\mathrm{Cl}\left(X_{\Delta}\right), \mathbb{k}^{\times}\right) \cong\left(\mathbb{k}^{\times}\right)^{|\mathscr{\Phi}|-\left(\begin{array}{c}n \\ 2\end{array}\right)+n}$.

(3) (VGIT) Given $\beta \in \mathbb{Z}^{n}$ there is $\alpha \in \mathbb{Z}^{|\mathscr{\Phi}|-\left(\begin{array}{c}n \\ 2\end{array}\right)+n}$ for which

$$
Z\left(I_{\bar{M}_{0, n}}\right) / / \alpha=G(2, n) / / \beta T^{n-1},
$$

so all GIT quotients of $G(2, n)$ by $T^{n-1}$ can be obtained from $\bar{M}_{0, n}$ by variation of the GIT.

Statement (3) relates to [Howard et al. 2009], where GIT quotients of $G(2, n)$ by $T^{n-1}$, or equivalently of $\left(\mathbb{P}^{1}\right)^{n}$ by $\operatorname{Aut}\left(\mathbb{P}^{1}\right)$, were studied.

Keel and Tevelev, in an article titled "Equations for $\bar{M}_{0, n}$ [2009], studied the image of the particular embedding of $\bar{M}_{0, n}$ into a product of projective spaces given by the complete linear series of the very ample divisor $\kappa=K_{\bar{M}_{0, n}}+\sum_{I \in \Phi} \delta_{I}$. Theorem 1.2 concerns projective embeddings of $\bar{M}_{0, n}$ corresponding to a full-dimensional subcone of the nef cone of $\bar{M}_{0, n}$, including that given by $\kappa$.

A key idea of this paper is to work in the Cox ring of sufficiently large toric subvarieties of $X_{\Sigma^{\star}}$. This often allows one to give equations in fewer variables. Also, a truly concrete description of $X_{\Sigma^{\star}}$ may be cumbersome or impossible, as in the case of $\bar{M}_{0, n}$, but a sufficiently large toric subvariety such as $X_{\Delta}$ can often be obtained. 
We now summarize the structure of the paper. Section 2 contains some tools from toric geometry that will be useful in the rest of the paper. The first part of Theorem 1.1 is proved in Section 3, while the GIT results are proved in Section 4. In Section 5 we explicitly describe the toric variety $X_{\Delta}$ that contains $\bar{M}_{0, n}$. In Sections 6 and 7 we prove Theorem 1.2. We end the paper with some natural questions about $\bar{M}_{0, n}$ arising from this work.

\section{Toric tools}

In this section we develop some tools to work with toric varieties that will be used in our applications to Chow and Hilbert quotients. We generally follow the notational conventions for toric varieties of [Fulton 1993], with the exception that we do not always require normality. Throughout $k$ is an algebraically closed field, and $\mathbb{k}^{\times}=\mathbb{k} \backslash\{0\}$. We denote by $T^{d}$ an algebraic torus isomorphic to $\left(\mathbb{k}^{\times}\right)^{d}$. If $I$ is an ideal in $\mathbb{k}\left[x_{0}, \ldots, x_{m}\right]$ then $Z(I)$ is the corresponding subscheme of either $\mathbb{A}^{m+1}$ or $\mathbb{P}^{m}$ depending on the context.

2A. Producing equations for quotients of subvarieties of tori. We first describe how to obtain equations for the quotient of a subvariety of a torus by a subtorus. Let $Y$ be a subscheme of a torus $T^{m}$ that is equivariant under a faithful action of $T^{d}$ on $T^{m}$ given by $(t \cdot x)_{j}=\left(\prod_{i=1}^{d} t_{i}^{a_{i j}}\right) x_{j}$, and let $I(Y) \subset \mathbb{k}\left[x_{1}^{ \pm 1}, \ldots, x_{m}^{ \pm 1}\right]$ be the ideal of $Y$. Write $A$ for the $d \times m$ matrix with $i j$-th entry $a_{i j}$. Let $D$ be a $(m-d) \times m$ matrix of rank $m-d$ whose rows generate the integer kernel of $A$, so $A D^{T}=0$. The matrix $D$ is a Gale dual for the $d \times m$ matrix $A=\left(a_{i j}\right)$ (see [Ziegler 1995, Chapter 6]).

Proposition 2.1. Let $\phi: \mathbb{k}\left[z_{1}^{ \pm 1}, \ldots, z_{m-d}^{ \pm 1}\right] \rightarrow \mathbb{k}\left[x_{1}^{ \pm 1}, \ldots, x_{m}^{ \pm 1}\right]$ be given by $\phi\left(z_{i}\right)=$ $\prod_{j=1}^{m} x_{j}^{D_{i j}}$. Then the ideal of $Y / T^{d}$ in the coordinate ring $\mathbb{k}\left[z_{1}^{ \pm 1}, \ldots, z_{m-d}^{ \pm 1}\right]$ of $T^{m} / T^{d}$ is given by $\phi^{-1}(I(Y))$. This is generated by polynomials $g_{1}, \ldots, g_{s}$ for which $I(Y)=\left\langle\phi\left(g_{1}\right), \ldots, \phi\left(g_{s}\right)\right\rangle$.

Proof. The coordinate ring of the quotient $Y / T^{d}$ is by definition the ring of invariants of $\mathbb{k}\left[x_{1}^{ \pm 1}, \ldots, x_{m}^{ \pm 1}\right] / I(Y)$ under the induced action of $T^{d}$. The $T^{d}$ action on $T^{m}$ gives a $\mathbb{Z}^{d}$-grading of $\mathbb{k}\left[x_{i}^{ \pm 1}\right]$ by setting $\operatorname{deg} x_{i}=\boldsymbol{a}_{i}$, where $\boldsymbol{a}_{i}$ is the $i$-th column of the matrix $A$. Since $T^{d}$ acts equivariantly on $Y$, the ideal $I(Y)$ is homogeneous with respect to this grading, so $\mathbb{k}\left[x_{i}^{ \pm 1}\right] / I(Y)$ is also $\mathbb{Z}^{d}$-graded. The ring of invariants is precisely the degree-zero part of this ring.

To prove that this is isomorphic to $k\left[z_{1}^{ \pm 1}, \ldots, z_{m-d}^{ \pm 1}\right] / \phi^{-1}(I(Y))$, we first define an automorphism of the torus $T^{m}$ so that $T^{d}$ is mapped to the subtorus having first $m-d$ coordinates equal to one. Choose any $m \times m$ integer matrix $U$ with determinant one whose first $d$ rows consist of the matrix $D$. This is possible because by the definition of $D$ the cokernel $\mathbb{Z}^{m} / \operatorname{im}\left(D^{T}\right)$ is torsion-free, so $\mathbb{Z}^{m} \cong \operatorname{im}\left(D^{T}\right) \oplus \mathbb{Z}^{d}$. 
Then the map $\tilde{\phi}: \mathbb{k}\left[z_{1}^{ \pm 1}, \ldots, z_{m}^{ \pm 1}\right] \rightarrow \mathbb{k}\left[x_{1}^{ \pm 1}, \ldots, x_{m}^{ \pm 1}\right]$ defined by $\tilde{\phi}\left(z_{i}\right)=\prod_{j=1}^{m} x_{j}^{U_{i j}}$ determines an automorphism of the torus $T^{m}$. Note that the map $\phi$ is $\tilde{\phi}$ restricted to the ring $\mathbb{k}\left[z_{1}^{ \pm 1}, \ldots, z_{m-d}^{ \pm 1}\right]$.

The ring $\mathbb{k}\left[z_{1}^{ \pm 1}, \ldots, z_{m}^{ \pm 1}\right]$ gets an induced $\mathbb{Z}^{d}$-grading from the grading on $\mathbb{k}\left[x_{j}^{ \pm 1}\right]$ by setting $\operatorname{deg} z_{i}=\sum_{j=0}^{m} U_{i j} \boldsymbol{a}_{j}$, which is the $i$-th column of $A U^{T}$. Since the first $m-d$ rows of $U$ are the rows of $D$, and $A D^{T}=0$, we thus have $\operatorname{deg} z_{i}=$ $0 \in \mathbb{Z}^{d}$ for $1 \leq i \leq m-d$. The degrees of the $d$ variables $z_{m-d+1}, \ldots, z_{m}$ are linearly independent, $\operatorname{since} \operatorname{rank}\left(A U^{T}\right)$ is $d$. This means that the degree zero part of $\mathbb{k}\left[z_{1}^{ \pm 1}, \ldots, z_{m}^{ \pm 1}\right]$ is $\mathbb{k}\left[z_{1}^{ \pm 1}, \ldots, z_{m-d}^{ \pm 1}\right]$, which proves that the coordinate ring of $Y / T^{d}$ is given by $\mathbb{k}\left[z_{1}^{ \pm 1}, \ldots, z_{m-d}^{ \pm 1}\right] / J$, where $J=\tilde{\phi}^{-1}(I(Y)) \cap \mathbb{k}\left[z_{1}^{ \pm 1}, \ldots, z_{m-d}^{ \pm 1}\right]$. The result then follows since $J=\phi^{-1}(I(Y))$. The statement about generators follows from the fact that $\phi$ is injective, since $\tilde{\phi}$ is an isomorphism.

Example 2.2. Let $Y$ be the subscheme of $T^{10}$ defined by the ideal

$$
\begin{aligned}
I= & \left\langle x_{12} x_{34}-x_{13} x_{24}+x_{14} x_{23}, x_{12} x_{35}-x_{13} x_{25}+x_{15} x_{23},\right. \\
& \left.x_{12} x_{45}-x_{14} x_{25}+x_{15} x_{24}, x_{13} x_{45}-x_{14} x_{35}+x_{15} x_{34}, x_{23} x_{45}-x_{24} x_{35}+x_{25} x_{34}\right\rangle \\
\subseteq & \mathbb{k}\left[x_{i j}^{ \pm 1}: 1 \leq i<j \leq 5\right] .
\end{aligned}
$$

This is the intersection with the torus of $A^{10}$ of the affine cone over the Grassmannian $G(2,5)$ in its Plücker embedding into $\mathbb{P}^{9}$. The torus $T^{5}$ acts equivariantly on $Y$ by $t \cdot x_{i j}=t_{i} t_{j} x_{i j}$, giving rise to matrices

$$
A=\left(\begin{array}{llllllllll}
1 & 1 & 1 & 1 & 0 & 0 & 0 & 0 & 0 & 0 \\
1 & 0 & 0 & 0 & 1 & 1 & 1 & 0 & 0 & 0 \\
0 & 1 & 0 & 0 & 1 & 0 & 0 & 1 & 1 & 0 \\
0 & 0 & 1 & 0 & 0 & 1 & 0 & 1 & 0 & 1 \\
0 & 0 & 0 & 1 & 0 & 0 & 1 & 0 & 1 & 1
\end{array}\right), \quad D=\left(\begin{array}{rrrrrrrrrr}
0 & 1 & -1 & 0 & -1 & 1 & 0 & 0 & 0 & 0 \\
0 & 1 & 0 & -1 & -1 & 0 & 1 & 0 & 0 & 0 \\
1 & 0 & -1 & 0 & -1 & 0 & 0 & 1 & 0 & 0 \\
1 & 0 & 0 & -1 & -1 & 0 & 0 & 0 & 1 & 0 \\
1 & 1 & -1 & -1 & -1 & 0 & 0 & 0 & 0 & 1
\end{array}\right),
$$

where the columns are ordered $\{12,13, \ldots, 35,45\}$. The map $\phi: \mathbb{k}\left[z_{1}^{ \pm 1}, \ldots, z_{5}^{ \pm 1}\right]$ $\rightarrow \mathbb{k}\left[x_{i j}^{ \pm 1}: 1 \leq i<j \leq 5\right]$ is given by $\phi\left(z_{1}\right)=x_{13} x_{24} / x_{14} x_{23}, \phi\left(z_{2}\right)=x_{13} x_{25} / x_{15} x_{23}$, $\phi\left(z_{3}\right)=x_{12} x_{34} / x_{14} x_{23}, \phi\left(z_{4}\right)=x_{12} x_{35} / x_{15} x_{23}$, and $\phi\left(z_{5}\right)=x_{12} x_{13} x_{45} / x_{14} x_{15} x_{23}$.

The ideal $\phi^{-1}(I)$ is then

$$
\begin{aligned}
\left\langle z_{3}-z_{1}+1, z_{4}-z_{2}\right. & \left.+1, z_{5}-z_{2}+z_{1}, z_{5}-z_{4}+z_{3}, z_{5}-z_{1} z_{4}+z_{2} z_{3}\right\rangle \\
& =\left\langle z_{3}-z_{1}+1, z_{4}-z_{2}+1, z_{5}-z_{2}+z_{1}\right\rangle \subset \mathbb{k}\left[z_{1}^{ \pm 1}, \ldots, z_{5}^{ \pm 1}\right] .
\end{aligned}
$$

For this it is essential that we work in the Laurent polynomial ring; for example, $\phi\left(z_{5}-z_{2}+z_{1}\right)=x_{13} /\left(x_{14} x_{15} x_{23}\right)\left(x_{12} x_{45}-x_{14} x_{25}+x_{15} x_{24}\right)$. The variety of $\phi^{-1}(I)$ is the moduli space $M_{0,5}$. Note that this shows that $M_{0,5}$ is a complete intersection in $T^{5}$, cut out by three linear equations. This example is continued in Example 3.1 and Sections 5, 6, and 7. 
2B. Producing equations for closures in toric varieties. The next proposition describes how to find the ideal of the closure of a subvariety of a torus in a toric variety. We use the notation $T^{m-d}$ for ease of connection with the rest of this section, but there is no requirement that this torus be obtained as a quotient.

Recall that the Cox ring of a normal toric variety $X_{\Sigma}$ (see [Cox 1995; Mustaţă 2002]) is the polynomial ring $S=\mathbb{k}\left[y_{1}, \ldots, y_{r}\right]$, where $r=|\Sigma(1)|$ is the number of rays of $\Sigma$. It is graded by the divisor class group of $X_{\Sigma}$, so that deg $y_{i}=\left[D_{i}\right]$, where $\left[D_{i}\right] \in \mathrm{Cl}\left(X_{\Sigma}\right)$ is the class of the torus-invariant divisor $D_{i}$ associated to the $i$-th ray $\rho_{i}$ of $\Sigma$. An ideal $I \subset S$ determines an ideal sheaf $\tilde{I}$ on $X_{\Sigma}$, and thus a closed subscheme of $X_{\Sigma}$, and conversely, every ideal sheaf on $X_{\Sigma}$ is of the form $\tilde{I}$ for some ideal $I$ of $S$ (Theorem 1.1 of [Mustaţă 2002] removes the need for the simplicial hypothesis in [Cox 1995, Theorem 3.7]). The sheaf $\tilde{I}$ is given on an affine chart $U_{\sigma}$ of $X_{\Sigma}$ by $I_{\sigma}=\left(I S_{\prod_{i \notin \sigma} y_{i}}\right)_{0}$. The correspondence between ideals in $S$ and closed subschemes of $X_{\Sigma}$ is not bijective, but for any closed subscheme $Z \subset X_{\Sigma}$ there is a largest ideal $I(Z) \subset S$ with $\widetilde{I(Z)}=g_{Z}$.

Recall also that if $I$ is an ideal in a ring $R$ and $y \in R$, then $\left(I: y^{\infty}\right)=\{r \in$ $R: r y^{k} \in I$ for some $\left.k>0\right\}$. Geometrically this removes irreducible components supported on the variety of $y$.

Proposition 2.3. Let $X_{\Sigma}$ be an $(m-d)$-dimensional toric variety with Cox ring $S=\mathbb{k}\left[y_{1}, \ldots, y_{r}\right]$. Set $y=\prod_{i=1}^{r} y_{i}$ so that

$$
\rho: \mathbb{k}\left[T^{m-d}\right]=\mathbb{k}\left[z_{1}^{ \pm 1}, \ldots, z_{m-d}^{ \pm 1}\right] \longrightarrow\left(S_{y}\right)_{0}
$$

is the isomorphism given by the inclusion of the torus $T^{m-d}$ into $X_{\Sigma}$. If $Y \subset T^{m-d}$ is given by ideal $I(Y)=\left\langle f_{1}, \ldots, f_{s}\right\rangle \subset \mathbb{k}\left[T^{m-d}\right]$, then the ideal I for the closure $\bar{Y}$ of $Y$ in $X_{\Sigma}$ is $\left(\rho(I(Y)) S_{y}\right) \cap S$, which is

$$
I=\left(\left\langle\widetilde{\rho\left(f_{i}\right)}: 1 \leq i \leq s\right\rangle: y^{\infty}\right),
$$

where $\widetilde{\rho\left(f_{i}\right)}$ is obtained by clearing the denominator of $\rho\left(f_{i}\right)$.

Proof. Let $J=\rho(I(Y)) \subset\left(S_{y}\right)_{0}$. The closure $\bar{Y}$ of $Y$ in $X_{\Sigma}$ is the smallest closed subscheme of $X_{\Sigma}$ containing $Y$. Since the torus $T^{m-d}$ is the affine toric variety corresponding to the cone $\sigma$ consisting of just the origin in $\Sigma$, for this $\sigma$ we have $I_{\sigma}=\left(I S_{y}\right)_{0}$ for any ideal $I \subseteq S$. As the correspondence between subschemes $Z$ of $X_{\Sigma}$ and ideals $I(Z)$ of $S$ is inclusion reversing, we have that $I(\bar{Y})$ is the largest ideal $I$ in $S$ with $\left(I S_{y}\right)_{0} \subseteq J$ for which $I=I(Z)$ for some subscheme $Z$ of $X_{\Sigma}$.

There is a monomial, and thus a unit, in any degree $a$ for which $\left(S_{y}\right)_{a}$ is nonzero, so if $K$ is an ideal in $S_{y}$, then $K_{0} S_{y}=K$, and $(K \cap S) S_{y}=K$. Thus ( $\left(J S_{y} \cap\right.$ $\left.S) S_{y}\right)_{0}=\left(J S_{y}\right)_{0}=J$, and if $I$ is any homogeneous ideal in $S$ with $\left(I S_{y}\right)_{0} \subset J$ then $I \subseteq I S_{y} \cap S \subset J S_{y} \cap S$. Thus to show that $I=J S_{y} \cap S$ is the ideal of $I(\bar{Y})$, we need only show that $I$ is of the form $I(Z)$ for some subscheme $Z$ of 
$X_{\Sigma}$. Indeed, let $I^{\prime}$ be the largest ideal in $S$ with $\tilde{I}^{\prime}=\tilde{I}$. Then by construction we have $\left(I^{\prime} S_{y}\right)_{0}=\left(I S_{y}\right)_{0}=J$, so by above $I^{\prime} \subseteq I$, and thus $I=I^{\prime}$. This shows that $I(\bar{Y})=J S_{y} \cap S$.

Suppose now that $I(Y)$ is generated by $\left\{f_{1}, \ldots, f_{s}\right\} \subset \mathbb{k}\left[z_{1}^{ \pm 1}, \ldots, z_{m-d}^{ \pm 1}\right]$. Then $J S_{y}$ is generated by $\left\{\rho\left(f_{1}\right), \ldots, \rho\left(f_{s}\right)\right\}$. The denominator of each $\rho\left(f_{i}\right)$ is a monomial, which is a unit in $S_{y}$, so $J S_{y}$ is also generated by the polynomials $\widetilde{\rho\left(f_{i}\right)}$ obtained by clearing the denominators in the $\rho\left(f_{i}\right)$. The result follows from the observation that if $K$ is an ideal in $S_{y}$, generated by $\left\{g_{1}, \ldots, g_{s}\right\} \subset S$, then $K \cap S=\left(\left\langle g_{1}, \ldots, g_{s}\right\rangle: y^{\infty}\right)$. See, for example, [Eisenbud 1995, Exercise 2.3].

2C. Sufficiently large subvarieties via tropical geometry. A key idea of this paper is to work in toric varieties whose fan has few cones. This is made precise in the following definition.

Definition 2.4. A toric subvariety $X_{\Delta}$ of a toric variety $X_{\Sigma}$ with torus $T^{m}$ is sufficiently large with respect to a subvariety $Y \subseteq X_{\Sigma}$ if the fan $\Delta$ contains all cones of $\Sigma$ corresponding to $T^{m}$-orbits of $X_{\Sigma}$ that intersect $Y$.

Tropical geometry provides the tools to compute whether a given toric subvariety $X_{\Delta} \subseteq X_{\Sigma}$ is sufficiently large for $Y \subseteq X_{\Sigma}$. We now review the version we use in this paper. Let $Y \subset T^{m} \cong\left(\mathbb{k}^{\times}\right)^{m}$ be a subscheme defined by the ideal $I=I(Y) \subset$ $S:=\mathbb{k}\left[x_{1}^{ \pm 1}, \ldots, x_{m}^{ \pm 1}\right]$. Given a vector $w \in \mathbb{R}^{m}$ we can compute the leading form $\operatorname{in}_{w}(f)$ of a polynomial $f \in S$, which is the sum of those terms $c_{u} x^{u}$ in $f$ with $w \cdot u$ minimal. The initial ideal $\operatorname{in}_{w}(J)$ is $\left\langle\operatorname{in}_{w}(f): f \in J\right\rangle$. Let $K$ be any algebraically closed field extension of $\mathbb{k}$ with a nontrivial valuation val $: K^{\times} \rightarrow \mathbb{R}$ such that $\operatorname{val}(\mathbb{k})=0$. We denote by $V_{K}(I)$ the set $\left\{u \in\left(K^{\times}\right)^{m}: f(u)=0\right.$ for all $\left.f \in I\right\}$.

Definition/Theorem 2.5. Let $Y$ be a subvariety of $T^{m}$. The tropical variety of $Y$, denoted $\operatorname{Trop}(Y)$, is the closure in $\mathbb{R}^{m}$ of the set

$$
\left\{\left(\operatorname{val}\left(u_{1}\right), \ldots, \operatorname{val}\left(u_{m}\right)\right) \in \mathbb{R}^{m}:\left(u_{1}, \ldots, u_{m}\right) \in V_{K}(J)\right\} .
$$

This equals the set

$$
\left\{w \in \mathbb{R}^{m}: \operatorname{in}_{w}(I) \neq\langle 1\rangle\right\} .
$$

There is a polyhedral fan $\Sigma$ whose support is $\operatorname{Trop}(Y)$.

Versions of this result appear in [Speyer and Sturmfels 2004, Theorem 2.1] and [Einsiedler et al. 2006; Draisma 2008; Jensen et al. 2008; Payne 2009]. We consider here only the "constant coefficient" case, where the coefficients of polynomials generating defining ideal $I$ live in $\mathbb{k}$, so have valuation zero. This guarantees there is the structure of a fan on $\operatorname{Trop}(Y)$, rather than a polyhedral complex. We note that we follow the conventions for tropical varieties as tropicalizations of usual varieties as in, for example, [Speyer and Sturmfels 2004; Gathmann 2006] rather than the more intrinsic definition used by [Mikhalkin 2006]. For readers familiar 
with these works we emphasize that we follow the min convention for the tropical semiring rather than the max convention of [Gathmann 2006].

The key result is the following fundamental lemma and its immediate corollary.

Lemma 2.6 [Tevelev 2007, Lemma 2.2]. Let $Y$ be a subvariety of the torus $T^{m}$, and let $\mathscr{F}$ be an l-dimensional cone in $\mathbb{Q}^{m}$ whose rays are spanned by part of a basis for $\mathbb{Z}^{m} \subseteq \mathbb{Q}^{m}$. Let $U_{\mathscr{F}}=\mathbb{A}^{l} \times\left(\mathbb{k}^{\times}\right)^{m-l}$ be the corresponding affine toric variety. Then the closure $\bar{Y}$ of $Y$ in $U_{\mathscr{F}}$ intersects the closed orbit of $U_{\mathscr{F}}$ if and only if the interior of the cone $\mathscr{F}$ intersects the tropical variety of $Y$.

Corollary 2.7. Let $Y$ be a subvariety of the torus $T^{m}$. Let $X_{\Sigma}$ be an $m$-dimensional toric variety with dense torus $T^{m}$ and fan $\Sigma \subseteq \mathbb{R}^{m}$. Let $\operatorname{Trop}(Y) \subseteq \mathbb{R}^{m}$ be the tropical variety of $Y \subseteq T^{m}$. Then the closure $\bar{Y}$ of $Y$ in $X_{\Sigma}$ intersects the $T^{m}$-orbit of $X_{\Sigma}$ corresponding to a cone $\sigma \subseteq \Sigma$ if and only if $\operatorname{Trop}(Y)$ intersects the interior of $\sigma$.

Thus a toric subvariety $X_{\Delta}$ of $X_{\Sigma}$ with $\Delta$ a subfan of $\Sigma$ is sufficiently large with respect to $\bar{Y}$ exactly when $\Delta$ contains every cone of $\Sigma$ whose relative interior intersects the $\operatorname{Trop}(Y)$.

Proof. Since $\Sigma$ is not assumed to be smooth, or even simplicial, we first resolve singularities. Let $\pi: X_{\Sigma^{\prime}} \rightarrow X_{\Sigma}$ be a toric resolution of singularities, so $\Sigma^{\prime}$ is a refinement of the fan $\Sigma$, and let $\bar{Y}^{\prime}$ be the strict transform of $\bar{Y}$ in $X_{\Sigma^{\prime}}$, which is the closure of $Y$ in $X_{\Sigma^{\prime}}$. It suffices to prove the corollary for $\bar{Y}^{\prime} \subseteq X_{\Sigma^{\prime}}$, as $\bar{Y}$ intersects the orbit corresponding to a cone $\sigma \in \Sigma$ if and only if $\bar{Y}^{\prime}$ intersects the orbit corresponding to a cone $\sigma^{\prime} \in \Sigma^{\prime}$ with $\sigma^{\prime} \subseteq \sigma$. Since the orbit corresponding to a cone $\sigma^{\prime} \in \Sigma^{\prime}$ is the closed orbit of the corresponding $U_{\sigma^{\prime}}$, this result now follows from Lemma 2.6.

Example 2.8. Let $\Sigma_{1}$ and $\Sigma_{2}$ be the two complete fans with rays as pictured in Figure 1. If $Y \subset T^{2}$ is a subvariety with tropicalization the dotted line shown in both figures, then the shaded fans define sufficiently large toric subvarieties with respect to the respective closures of $Y$ in $X_{\Sigma_{1}}$ and $X_{\Sigma_{2}}$.
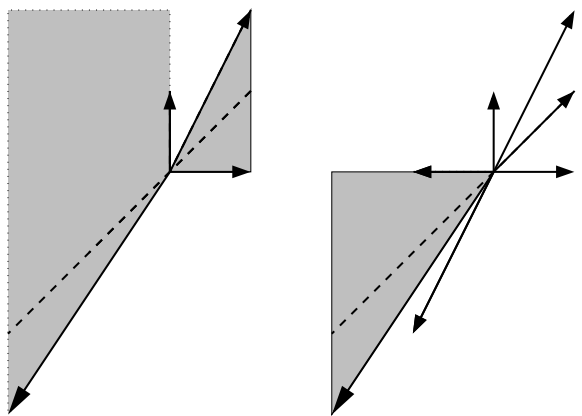

Figure 1. Sufficient toric subvarieties of $X_{\Sigma}$ with respect to $\bar{Y}$. 


\section{Equations for Chow and Hilbert quotients}

Given equations for a $T^{d}$-equivariant subscheme $X \subset \mathbb{P}^{m}$, we show in this section how to effectively compute generators for the ideal of the Chow or Hilbert quotient in the Cox ring of a suitably chosen toric variety.

3A. Definition of Chow and Hilbert quotients. We first recall the definition of the Chow and Hilbert quotients $X / /{ }^{\mathrm{Ch}} T^{d}$ and $X /{ }^{\mathrm{H}} T^{d}$ of a projective variety by the action of a $d$-dimensional algebraic torus $T^{d}$. We assume that $X$ is equivariantly embedded into a projective space $\mathbb{P}^{m}$ with no irreducible component contained in a coordinate subspace.

Identifying $T^{d}$ with $\left(\mathbb{k}^{\times}\right)^{d+1} /\left(\mathbb{k}^{\times}\right)$so that points in $T^{d}$ are equivalence classes $t=\left[t_{0}: \cdots: t_{d}\right]$, we can write

$$
T^{d} \times \mathbb{P}^{m} \rightarrow \mathbb{P}^{m}, \quad\left(t,\left[x_{0}, \ldots, x_{m}\right]\right) \mapsto\left[\left(\prod_{j=0}^{d} t_{j}^{a_{j 0}}\right) x_{0}, \ldots,\left(\prod_{j=0}^{d} t_{j}^{a_{j m}}\right) x_{m}\right],
$$

and let $A$ be the $(d+1) \times(m+1)$ matrix with $i j$-th entry $a_{i j}$ for $0 \leq i \leq d$ and $0 \leq j \leq m$. We assume that the $T^{d}$-action on $\mathbb{P}^{m}$ is faithful, so $A$ has rank $d+1$. The compatibility of the action with the diagonal $\mathbb{k}^{\times}$action on $\left(\mathbb{k}^{\times}\right)^{d+1}$ and $\left(\mathbb{k}^{\times}\right)^{m+1}$ means that all column sums of $A$ agree, so the row space of $A$ contains the allones vector. Let $X_{A} \subset \mathbb{P}^{m}$ be the closure of the $T^{d}$-orbit of $e=[1: \cdots: 1] \in T^{m}$. Then $X_{A}$ is a toric variety with associated torus $T^{d}$ and corresponding toric ideal $I_{A}=\left\langle x^{u}-x^{v}: A u=A v\right\rangle \subset \mathbb{k}\left[x_{0}, \ldots, x_{m}\right]$ (see [Gel'fand et al. 1994, Chapter 5] and [Sturmfels 1996, Chapter 4]).

We identify $T^{m}$ with the quotient $\left(\mathbb{k}^{\times}\right)^{m+1} /\left(\mathbb{k}^{\times}\right)$, so a point on $T^{m}$ is an equivalence class $s=\left[s_{0}: \cdots: s_{m}\right]$. Then $T^{m}$ acts on $\mathbb{P}^{m}$ by $s \cdot x=\left[s_{0} x_{0}: \cdots\right.$ : $\left.s_{m} x_{m}\right]$ and every point in the geometric quotient $T^{m} / T^{d}$ corresponds to an orbit of $T^{d}$ whose closure in $\mathbb{P}^{m}$ is a $d$-dimensional subscheme of $\mathbb{P}^{m}$ having ideal $I_{s A}=\left\langle s^{v} x^{u}-s^{u} x^{v}: A u=A v\right\rangle \subset \mathbb{k}\left[x_{0}, \ldots, x_{m}\right]$, with $s \in T^{m}$. These orbit closures all have the same Hilbert polynomial, and so define closed points on the same connected component of $\operatorname{Hilb}\left(\mathbb{P}^{m}\right)$, and there is an induced morphism $\phi_{H}: T^{m} / T^{d} \rightarrow \operatorname{Hilb}\left(\mathbb{P}^{m}\right)$. The Hilbert quotient $X /{ }^{\mathrm{H}} T^{d}$ is defined to be the closure in $\operatorname{Hilb}\left(\mathbb{P}^{m}\right)$ of $\phi_{H}\left(\left(X \cap T^{m}\right) / T^{d}\right)$. Since $\mathbb{P}^{m} / /^{\mathrm{H}} T^{d}$ is the closure of $\phi_{H}\left(T^{m} / T^{d}\right)$ in $\operatorname{Hilb}\left(\mathbb{P}^{m}\right), X / /{ }^{\mathrm{H}} T^{d} \subset \mathbb{P}^{m} /{ }^{\mathrm{H}} T^{d}$.

Analogously, there is a morphism $\phi_{\mathrm{Ch}}: T^{m} / T^{d} \rightarrow \operatorname{Chow}\left(\mathbb{P}^{m}\right)$ (see [Kollár 1996, Sections 1.3 and 1.4]). The Chow quotient $X / /^{\mathrm{Ch}} T^{d}$ is defined to be the closure in Chow $\left(\mathbb{P}^{m}\right)$ of $\phi_{\mathrm{Ch}}\left(\left(X \cap T^{m}\right) / T^{d}\right)$ and $X /{ }^{\mathrm{Ch}} T^{d} \subset \mathbb{P}^{m} / /^{\mathrm{Ch}} T^{d}$.

We remark that while the definitions given here appear to depend on the choice of projective embedding, the Chow and Hilbert quotients of $X$ are in fact independent of this choice. See [Kapranov 1993] for a more intrinsic formulation. See also [Białynicki-Birula and Sommese 1987] for original work on the Hilbert quotient. 
For the most part the Chow and Hilbert quotients can be treated uniformly, and we use the notation $X / /^{\star} T^{d}$ to denote either of $X / /{ }^{\mathrm{Ch}} T^{d}$ or $X / /{ }^{\mathrm{H}} T^{d}$.

The following example will be developed more in Section 5.

Example 3.1. Consider the action of the $(n-1)$-dimensional torus

$$
T^{n-1} \cong\left(\mathbb{k}^{\times}\right)^{n} / \mathbb{k}^{\times}
$$

on $\mathbb{P}_{\mathbb{C}}^{\left(\begin{array}{c}n \\ 2\end{array}\right)-1}$ given by

$$
\left[t_{1}: \cdots: t_{n}\right] \cdot\left[\left\{x_{i j}\right\}_{1 \leq i<j \leq n}\right]=\left[\left\{t_{i} t_{j} x_{i j}\right\}_{1 \leq i<j \leq n}\right] .
$$

Recall that the Plücker embedding of the Grassmannian $G(2, n)$ into $\mathbb{P}^{\left(\begin{array}{l}n \\ 2\end{array}\right)-1}$ is given by taking a subspace $V$ to $V \wedge V$, or by taking a $2 \times n$ matrix representing a choice of basis for $V$ to its vector of $2 \times 2$ minors. The ideal $I_{2, n}$ of $G(2, n)$ in the homogeneous coordinate ring of $\mathbb{P}\left(\begin{array}{l}n \\ 2\end{array}\right)-1$ is generated by the set of Plücker equations $\left\langle p_{i j k l}=x_{i j} x_{k l}-x_{i k} x_{j l}+x_{i l} x_{j k}: 1 \leq i<j<k<l \leq n\right\rangle$ and hence is $T^{n-1}$-equivariant. Let $G^{0}(2, n)$ be the open set inside $G(2, n)$ consisting of those points with nonvanishing Plücker coordinates, corresponding to those two-planes that do not have a nonzero intersection with any two coordinate hyperplanes. The torus $T^{n-1}$ acts freely on $G^{0}(2, n)$ and all orbits have maximal dimension. The moduli space $M_{0, n}$ parametrizing smooth $n$-pointed rational curves is equal to the geometric quotient $G^{0}(2, n) / T^{n-1}$. By [Kapranov 1993, Theorem 4.1.8]

$$
G(2, n) / /{ }^{\mathrm{Ch}} T^{n-1}=G(2, n) /{ }^{\mathrm{H}} T^{n-1}=\bar{M}_{0, n} .
$$

3B. Chow and Hilbert quotients of projective spaces. The Chow and the Hilbert quotients of $\mathbb{P}^{m}$ by $T^{d}$ are both not necessarily normal toric varieties [Kapranov et al. 1992]. We next describe the fans $\Sigma^{\mathrm{Ch}}$ and $\Sigma^{\mathrm{H}}$ associated to the normalizations of $\mathbb{P}^{m} / /{ }^{\mathrm{Ch}} T^{d}$ and $\mathbb{P}^{m} / /{ }^{\mathrm{H}} T^{d}$. The fan $\Sigma^{\mathrm{Ch}}$ is the secondary fan of the matrix $A$ given in (1). Top-dimensional cones of the secondary fan correspond to regular triangulations of the vector configuration determined by the columns of $A$. These are also indexed by radicals of initial ideals of $I_{A}$ by [Sturmfels 1996, Theorem 8.3]. See [Gel'fand et al. 1994] for a description of the secondary fan. The fan $\Sigma^{\mathrm{H}}$ is the saturated Gröbner fan, whose cones are indexed by the saturation of initial ideals of $I_{A}$ with respect to the irrelevant ideal $\left\langle x_{0}, \ldots, x_{m}\right\rangle$. See [Bayer and Morrison 1988; Mora and Robbiano 1988] for the original work on the Gröbner fan, and [Sturmfels 1996; Maclagan and Thomas 2007] for expositions. When we want to refer to both fans simultaneously we use the notation $\Sigma^{\star}$.

We denote by $\bar{N}$ the common lattice of the fans $\Sigma^{\mathrm{Ch}}$ and $\Sigma^{\mathrm{H}}$. Note that $\bar{N}=$ $N / N^{\prime}$, where $N \cong \mathbb{Z}^{m+1}$ and $N^{\prime}$ is the integer row space of the matrix $A$. The torus $T^{d+1}$ with $T^{d}=T^{d+1} / \mathbb{k}^{\times}$is equal to $N^{\prime} \otimes \mathbb{k}^{\times}$, and $T^{m-d}=T^{m+1} / T^{d+1} \cong \bar{N} \otimes \mathbb{k}^{\times}$.

The images of the basis elements $\boldsymbol{e}_{i} \in \mathbb{Z}^{m+1} \cong N$ correspond to rays of $\Sigma^{\star}$. We can thus identify these rays with the columns of the Gale dual $D$ of the matrix 
$A$ (see Section 2A). By the construction of $D$ the integer column space of $D$ is $\mathbb{Z}^{m-d} \cong \bar{N}$.

3C. Equations for Chow and Hilbert quotients. In this section we show how to give equations for the Chow or Hilbert quotient of $X$ in the Cox ring of a toric variety $X_{\Sigma}$. Throughout this section $X$ is a $T^{d}$-equivariant subscheme of $\mathbb{P}^{m}$ with no irreducible component lying in any coordinate subspace, and we choose $X_{\Sigma}$ to be a sufficiently large toric subvariety of the normalization $X_{\Sigma^{\star}}$ of $\mathbb{P}^{m} / /^{\star} T^{d}$ with respect to the pullback of $X / /^{\star} T^{d}$ to $X_{\Sigma^{\star}}$. We recall that the notation $X / /{ }^{\star} T^{d}$ stands for either the Chow or Hilbert quotient, and any statement using this notation is short-hand for two separate results; one for the Chow quotient, and one for the Hilbert quotient. We denote by $I(X) \subseteq \mathbb{k}\left[x_{0}, \ldots, x_{m}\right]$ the saturated ideal defining $X$. Let the $(d+1) \times(m+1)$ matrix $A$ record the weights of the $T^{d}$ action, and let $D$ be its Gale dual (see (1)). Let $R$ be the matrix whose columns are the first integer lattice points on the rays of $\Sigma$. Since the columns of $D$ span the lattice $\bar{N}$ of $\Sigma^{\star}$, one has that

$$
R=D V,
$$

for some $(m+1) \times r$ matrix $V$. We denote by $X / /_{n}^{\star} T^{d}$ the pullback of $X / /^{\star} T^{d}$ to the normalization $X_{\Sigma^{\star}}$ of $\mathbb{P}^{m} / /{ }^{\star} T^{d}$.

Theorem 3.2. Let $T^{d}$ act on $\mathbb{P}^{m}$ and let $X \subset \mathbb{P}^{m}$ be a $T^{d}$-equivariant subscheme with $I(X)=\left\langle f_{1}, \ldots, f_{g}\right\rangle \subseteq \mathbb{k}\left[x_{0}, \ldots, x_{m}\right]$. Let $X_{\Sigma}$ be a toric subvariety of $X_{\Sigma^{\star}}$ containing $X / \|_{n}^{\star} T^{d}$, and let $S=\mathbb{k}\left[y_{1}, \ldots y_{r}\right]$ be the Cox ring of $X_{\Sigma}$. Let

$$
v: \mathbb{k}\left[x_{0}, \ldots, x_{m}\right] \rightarrow \mathbb{k}\left[y_{1}^{ \pm 1}, \ldots, y_{r}^{ \pm 1}\right] \quad \text { be given by } v\left(x_{i}\right)=\prod_{j=1}^{r} y_{j}^{V_{i j}} .
$$

The ideal of $X / /_{n}^{\star} T^{d}$ in $S$ is $I=v(I(X)) \cap S$, and is obtained by clearing denominators in $\left\{v\left(f_{i}\right): 1 \leq i \leq g\right\}$, and then saturating the result by the product of all the variables in $S$. If $X / /^{\star} T^{d}$ is irreducible and normal, it is isomorphic to $X / /{ }_{n}^{\star} T^{d}$ and $I$ is the ideal of $X / /^{\star} T^{d}$ in $S$.

Remark 3.3. When $X_{\Sigma}$ contains all rays of $\Sigma^{\star}$ corresponding to columns of $D$, and $D$ has no repeated columns, we can write $V=\left(I \mid C^{T}\right)$, where $I$ is the $(m+1) \times$ $(m+1)$ identity matrix and $C$ is an integer $(r-m-1) \times(m+1)$ matrix. Thus the map $v$ may be thought of as homogenizing the ideal $I(X)$ with respect to the grading of $S$. This is the formulation assumed in Theorem 1.1(1).

Proof of Theorem 3.2. The proof of the first claim proceeds in three parts. First, using Proposition 2.1, we find equations in $\mathbb{k}\left[z_{1}^{ \pm 1}, \ldots, z_{m-d}^{ \pm 1}\right]$, the coordinate ring of the torus $T^{m} / T^{d}$ of $X_{\Sigma}$, for $\left(X \cap T^{m}\right) / T^{d}$. Second, we use the isomorphism $\rho: \mathbb{k}\left[z_{1}^{ \pm 1}, \ldots, z_{m-d}^{ \pm 1}\right] \rightarrow S_{\left(\Pi_{i=1}^{r} y_{i}\right)}$, and apply Proposition 2.3 to obtain generators for the ideal in $S$ of the closure of $\left(X \cap T^{m}\right) / T^{d}$ in $X_{\Sigma^{\star}}$. Since this is $X / /_{n}^{\star} T^{d}$, we 
last check that this is the same as the ideal obtained by clearing denominators and saturating.

The key diagram is the following, where $U$ is as in the proof of Proposition 2.1 and the $i$ are inclusions:

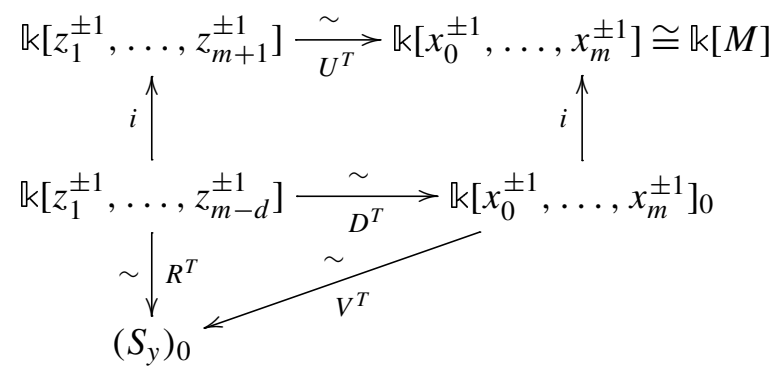

The content of Proposition 2.1 is that the ideal in $\mathbb{k}\left[z_{1}^{ \pm 1}, \ldots, z_{m-d}^{ \pm 1}\right]$ of $\left(X \cap T^{m}\right) / T^{d}$ is given by $i^{-1}\left(U^{T^{-1}}(I(X))\right)$, which is well-defined since $U^{T}(I(X))$ is in the image of $i$. The ideal $i^{-1}\left(U^{T}-1(I(X))\right)$ is taken under the map $D^{T}$ to the degree zero part of the ideal $I(X)$ in $\mathbb{k}\left[x_{0}^{ \pm 1}, \ldots, x_{m}^{ \pm 1}\right]$, since $U$ is chosen in Proposition 2.1 to have its first $d+1$ rows equal to $D$. We thus see that the choice of identification of the lattice $\bar{N}$ of the fan $\Sigma$ with $\mathbb{Z}^{m-d}$ identifies $\mathbb{k}[\bar{M}]$ with $\mathbb{k}\left[z_{1}^{ \pm 1}, \ldots, z_{m-d}^{ \pm 1}\right]$, where $\bar{M}$ is the lattice dual to $\bar{N}$. This means that the isomorphism $\mathbb{k}[\bar{M}] \cong\left(S_{y}\right)_{0}$ given in [Cox 1995, Lemma 2.2] is given by the matrix $R^{T}$, so the function $\rho$ of Proposition 2.3 is given by $\rho\left(z_{i}\right)=\prod_{j=1}^{r} y_{j}^{R_{i j}}$. It thus follows from Proposition 2.3 that the ideal in $S$ of the closure of $\left(X \cap T^{m}\right) / T^{d}$ in $X_{\Sigma}$ is given by applying $R^{T} \circ i^{-1} \circ U^{T^{-1}}$ to the generators of $I(X)$, clearing denominators, and then saturating by the product of the variables of $S$.

To complete the proof, it thus suffices to observe that $R^{T} \circ i^{-1} \circ U^{T^{-1}}$ restricted to the degree zero part of $\mathbb{k}\left[x_{0}^{ \pm 1}, \ldots, x_{m}^{ \pm 1}\right]$ is given by the matrix $V^{T}$. This follows from the fact that $R^{T}=V^{T} D^{T}$ and the bottom triangle of the above commutative diagram is made of three isomorphisms.

We now consider the case where $X / /{ }^{\star} T^{d}$ is irreducible and normal, and show that it isomorphic to $X / /{ }_{n}^{\star} T^{d}$. Set $Y=X / /^{\star} T^{d}$, and $Z=\mathbb{P}^{m} / /^{\star} T^{d}$. Let $\tilde{Y}$ and $\tilde{Z}=X_{\Sigma^{\star}}$ denote the respective normalizations. Begin by reducing to the case where $Z=\operatorname{Spec}(A)$ and $Y=\operatorname{Spec}(A / I)$ are irreducible affine schemes with $I$ an ideal in a ring $A$. Note that $Y$ intersects the torus $T^{m-d}$ of the not necessarily normal toric variety $Z$, and thus intersects the smooth locus of $Z$. Since $Y$ is irreducible, this means that the map $Y \times_{Z} \tilde{Z} \rightarrow Y$ is birational, and so $A$ and $A / I$ have the same total quotient ring. Normalization is a finite morphism, and the pullback of a finite morphism is finite, so $\tilde{A} / I \tilde{A}$ is integral over $A / I$. The isomorphism $\tilde{A} / I \tilde{A} \cong A / I$ then follows from the fact that $Y$ is normal, so $A / I$ is integrally closed. Finally since all maps are inclusions, everything glues to prove $Y \times_{Z} \tilde{Z} \cong Y$. 
Remark 3.4. The choice of the matrix $V$ in Theorem 3.2 is not unique. This does not affect the computation, however, as the induced map $v$ is unique when restricted to the degree zero part of $\mathbb{k}\left[x_{0}^{ \pm 1}, \ldots, x_{m}^{ \pm 1}\right]$.

Example 3.5. Theorem 3.2 lets us compute equations for $\bar{M}_{0,5}$. Kapranov's description of $\bar{M}_{0,5}$ as the Chow or Hilbert quotient of the Grassmannian $G(2,5)$ by the $T^{4}$ action, described in Example 3.1, gives embeddings of $\bar{M}_{0,5}$ into the normalizations of $\mathbb{P}^{9} / /{ }^{\mathrm{Ch}} T^{4}$ and $\mathbb{P}^{9} / /{ }^{\mathrm{H}} T^{4}$. These are both five-dimensional toric varieties whose rays include the columns of the matrix $D$ of Example 2.2, plus ten additional rays, being the columns of the matrix $-D$. Let $\Delta$ be the two-dimensional fan with rays the columns $\boldsymbol{d}_{i j}$ of $D$, and cones $\operatorname{pos}\left(\boldsymbol{d}_{i j}, \boldsymbol{d}_{k l}:\{i, j\} \cap\{k, l\}=\varnothing\right)$. By direct computation, or by Theorem 5.7, the fan $\Delta$ is a subfan of the fan of both $\Sigma^{\mathrm{Ch}}$ and $\Sigma^{\mathrm{H}}$ that defines a sufficiently large toric subvariety. For this fan we have $R=D$, so $V$ is the $\left(\begin{array}{c}10 \\ 2\end{array}\right) \times\left(\begin{array}{c}10 \\ 2\end{array}\right)$ identity matrix.

The ideal in $\mathbb{k}\left[x_{i j}: 1 \leq i<j \leq 5\right]$ defining $G(2,5)$ as a subscheme of $\mathbb{P}^{9}$ is generated by the Plücker equations given in Example 2.2. The Cox ring of $\mathbb{P}_{\Delta}$ is $\mathbb{k}\left[y_{i j}: 1 \leq i<j \leq 5\right]$, and the map $v$ of Theorem 3.2 is the identity map $v\left(x_{i j}\right)=y_{i j}$. The ideal of the normal irreducible variety $\bar{M}_{0,5}$ in the Cox ring of $\mathbb{P}_{\Delta}$ is thus generated by the Plücker relations. The saturation step is unnecessary in this case as this ideal is prime.

\section{GIT constructions of Chow/Hilbert quotients}

In this section we give a GIT construction of the Chow and Hilbert quotients of $X$. This follows from their description in the Cox ring of a toric variety in Section 3. We also recover all GIT quotients of $X$ by $T^{d}$ by variation of the GIT quotient. As before $X$ is a $T^{d}$-equivariant subscheme of $\mathbb{P}^{m}$ with no irreducible component lying in any coordinate hyperplane.

Let $X_{\Sigma}$ be a sufficiently large toric subvariety of $X_{\Sigma^{\star}}$. We assume in addition that $X_{\Sigma}$ has a torsion-free divisor class group, which can be guaranteed, for example, by taking $X_{\Sigma}$ to contain the rays of $\Sigma^{\star}$ corresponding to columns of the matrix $D$ (see Section 2A). Let $I$ be the ideal of $X / \|_{n}^{\star} T^{d}$ in the Cox ring of $X_{\Sigma}$. We next define a cone which will index our choices of GIT quotient.

Definition 4.1. Let $\left\{\left[D_{i}\right]: 1 \leq i \leq|\Sigma(1)|\right\}$ be the set of classes of the torus invariant divisors on $X_{\Sigma}$. Set

$$
\mathscr{G}\left(X_{\Sigma}\right)=\bigcap_{\sigma \in \Sigma} \operatorname{pos}\left(\left[D_{i}\right]: i \notin \sigma\right)
$$

We note that $\mathscr{G}\left(X_{\Sigma}\right)$ is the cone in $\mathrm{Cl}\left(X_{\Sigma}\right) \otimes \mathbb{R}$ spanned by divisor classes $[D] \in \mathrm{Cl}\left(X_{\Sigma}\right)$ for which $[D]$ is globally generated.

Lemma 4.2. The cone $\mathscr{G}\left(X_{\Sigma}\right)$ has positive dimension. 
Proof. Since $\Sigma$ is a subfan of the fan $\Sigma^{\star}$ we can number the rays of $\Sigma^{\star}$ so that the first $p$ live in $\Sigma$, while the last $s$ do not. If $i: X_{\Sigma} \rightarrow X_{\Sigma^{\star}}$ is the inclusion map, then the pullback from $\mathrm{Cl}\left(X_{\Sigma^{\star}}\right)$ to $\mathrm{Cl}\left(X_{\Sigma}\right)$ is given by $i^{\star}\left(\left[\sum_{i=1}^{p+s} a_{i} D_{i}\right]\right)=\left[\sum_{i=1}^{p} a_{i} D_{i}\right]$. Since $X_{\Sigma^{\star}}$ is a projective toric variety, its nef cone $\mathscr{G}\left(X_{\Sigma^{\star}}\right)=\bigcap_{\sigma \in \Sigma^{\star}} \operatorname{pos}\left(\left[D_{i}\right]\right.$ : $i \notin \sigma)$ is a positive-dimensional cone that can be viewed as living in $\mathrm{Cl}\left(X_{\Sigma^{\star}}\right) \otimes \mathbb{R}$. The image of $\mathscr{G}\left(X_{\Sigma^{\star}}\right)$ under the map $i^{*}$ is contained in $\mathscr{G}\left(X_{\Sigma}\right)$. We now show that some nonzero vector in $\mathscr{G}\left(X_{\Sigma^{\star}}\right)$ is taken to a nonzero vector under $i^{*}$. Indeed, otherwise every element of $\mathscr{G}\left(X_{\Sigma^{\star}}\right)$ could be written in the form $\sum_{i=p+1}^{p+s} a_{i}\left[D_{i}\right]$, so $\mathscr{G}\left(X_{\Sigma^{\star}}\right) \subseteq \operatorname{pos}\left(\left[D_{i}\right]: p+1 \leq i \leq s\right)$. But by the relation between the cones of $\mathscr{G}\left(X_{\Sigma^{\star}}\right)$ and chambers of the chamber complex [Billera et al. 1990], this means that the cone spanned by all the rays of $\Sigma$ lies in $\Sigma^{\star}$. However $X_{\Sigma}$ was assumed to be sufficiently large, which means that $\Sigma$ contains all cones of $\Sigma^{\star}$ intersecting the tropical variety of $X$, so the balancing condition on tropical varieties [Speyer 2005, Theorem 2.5.1] implies that the rays of $\Sigma$ positively span the entire space $\mathbb{R}^{m-d}$. This would mean that the cone spanned by the rays of $\Sigma$ was all of $\mathbb{R}^{m-d}$, contradicting it lying in the fan $\Sigma^{\star}$. We thus conclude that there are elements $v \in \mathscr{G}\left(X_{\Sigma^{\star}}\right)$ with $i^{*}(v) \neq 0$, so $\mathscr{G}\left(X_{\Sigma}\right)$ is a positive-dimensional cone.

Let $l=|\Sigma(1)|-\operatorname{dim}\left(X_{\Sigma}\right)$ be the rank of $\operatorname{Cl}\left(X_{\Sigma}\right)$. Let $H$ be the algebraic torus $\operatorname{Hom}\left(\mathrm{Cl}\left(X_{\Sigma}\right), \mathbb{k}^{\times}\right) \cong\left(\mathbb{k}^{\times}\right)^{l}$. We can regard $\mathrm{Cl}\left(X_{\Sigma}\right) \otimes \mathbb{R}$ as the space of (real) characters of the torus $H$, and $\mathscr{G}\left(X_{\Sigma}\right)$ as a subcone of the character space. The torus $H$ acts on $\mathbb{A}^{r}$ by $h \cdot x_{i}=h\left(\left[D_{i}\right]\right) x_{i}$. Recall that the torus of $X_{\Sigma}$ is $T^{m} / T^{d}$. We denote by relint $\left(G\left(X_{\Sigma}\right)\right)$ the relative interior of the cone $\mathscr{G}\left(X_{\Sigma}\right)$.

Let $r=|\Sigma(1)|$. Recall that for $\alpha \in \mathbb{Z}^{l}$ the GIT quotient $Y / / \alpha H$ of an affine variety $Y \subset \mathbb{A}^{r}$ is

$$
Y / / \alpha=\operatorname{Proj}\left(\bigoplus_{j \geq 0}\left(\mathbb{k}\left[x_{1}, \ldots, x_{r}\right] / I(Y)\right)_{j \alpha}\right),
$$

where the $\mathbb{Z}^{l}$ grading on the polynomial ring comes from the $H$-action on $\mathbb{A}^{r}$. For $\alpha \in \mathbb{Q}^{l}$ we define $Y / / \alpha H$ to be $Y / / s \alpha$ for any integral multiple $s \alpha$.

Proposition 4.3. Let $Y \subseteq \mathbb{A}^{r}$ be the subscheme defined by the ideal $I \subseteq \operatorname{Cox}\left(X_{\Sigma}\right)$ of $X / /_{n}^{\star} T^{d}$. For rational $\alpha \in \operatorname{relint}\left(G\left(X_{\Sigma}\right)\right)$ we have

$$
X / \|_{n}^{\star} T^{d}=Y / / \alpha H .
$$

Proof. It follows from the results of [Cox 1995] and the chamber complex description of the secondary fan that $X_{\Sigma^{\prime}}=\mathbb{A}^{r} / / \alpha H$ is a projective toric variety whose fan $\Sigma^{\prime}$ has same rays as $\Sigma$ and contains $\Sigma$ as a subfan. The dense torus of $X_{\Sigma^{\prime}}$ is also $T^{m} / T^{d}$. The quotient $Y / / \alpha H$ is a subvariety of $X_{\Sigma^{\prime}}$. Let $(Y / / \alpha H)^{0}=$ $(Y / / \alpha H) \cap T^{m} / T^{d}=\left(Y \cap T^{r}\right) / H=\left(X \cap T^{m}\right) / T^{d}$, where the last equality follows from the fact that $T^{r} / H \cong T^{m} / T^{d}$. Then $Y / / \alpha H$ is the closure of $(Y / / \alpha H)^{0}$ in $X_{\Sigma^{\prime}}$. By Corollary 2.7, to show that $Y / / \alpha H$ is the closure of $(Y / / \alpha H)^{0}$ inside $X_{\Sigma}$ it suffices to show that the tropical variety of $\left(X \cap T^{m}\right) / T^{d} \subseteq T^{m} / T^{d}$ is contained 
in the support of $\Sigma$. This follows from the hypothesis that $X_{\Sigma}$ is sufficiently large, again by Corollary 2.7. Since $X / /_{n}^{\star} T^{d}$ is the closure of $\left(X \cap T^{m}\right) / T^{d}$ in $X_{\Sigma}$, the proposition follows.

This GIT description gives projective embeddings of $X /{ }_{n}^{\star} T^{d}$, as we now describe. Let $S=\mathbb{k}\left[x_{1}, \ldots, x_{r}\right]$ be the Cox ring of $X_{\Sigma}$. For $\alpha \in \mathscr{G}\left(X_{\Sigma}\right)$, write $S^{\alpha}$ for the subring $\bigoplus_{j \geq 0} S_{j \alpha}$ of $S$. Note that $S^{\alpha}$ has a standard $\mathbb{Z}$-grading, by setting $\operatorname{deg} f=j$ for all $f \in S_{j \alpha}$. If $J$ is an ideal in $S$, write $J^{\alpha}$ for the ideal $J \cap S^{\alpha}$ of $S^{\alpha}$. The GIT description above gives that

$$
X / /{ }_{n}^{\star} T^{d}=\operatorname{Proj}\left(S^{\alpha} / I^{\alpha}\right) .
$$

Corollary 4.4. The GIT description of $X / \|_{n}^{\star} T^{d}$ gives a projective embedding of $X / /{ }_{n}^{\star} T^{d}$ with the pullback of $\mathcal{O}(1)$ on $\mathbb{P}^{N}$ equal to $\pi^{*}(\alpha)$ for a large enough multidegree $\alpha \in G\left(X_{\Sigma}\right)$, where $\pi$ is the embedding of $X / /{ }_{n}^{\star} T^{d}$ into $X_{\Sigma}$.

Proof. Assume $\alpha$ is large enough so that $S^{\alpha} / I^{\alpha}$ is generated in degree one. If not, we can replace $\alpha$ by $l \alpha$ for $l \gg 0$. Let $x^{u_{0}}, \ldots, x^{u_{N}} \in S_{\alpha}$ generate $S^{\alpha} / I^{\alpha}$, and define a map $\phi: \mathbb{k}\left[z_{0}, \ldots, z_{N}\right] \rightarrow S^{\alpha} / I^{\alpha}$ by $\phi\left(z_{i}\right)=x^{u_{i}}$. Let $J=\operatorname{ker} \phi$. Then $X / /{ }_{n}^{\star} T^{d}=\operatorname{Proj}\left(\mathbb{k}\left[z_{0}, \ldots, z_{N}\right] / J\right)$, and by construction the pullback of $\mathscr{O}(1)$ is $\pi^{*}(\alpha)$.

Example 4.5. We continue the discussion of $\bar{M}_{0,5}$ begun in Examples 3.1 and 3.5. Example 3.5 shows that $\bar{M}_{0,5}$ is the GIT quotient of the affine cone over the Grassmannian $G(2,5)$ by a five-dimensional torus, so $\bar{M}_{0,5}=G(2,5) / / \alpha T^{4}$ for all $\alpha$ in the relative interior of $\mathscr{G}\left(X_{\Delta}\right)$. The cone $\mathscr{G}\left(X_{\Delta}\right)$ is the intersection of the cones $\operatorname{pos}\left(\boldsymbol{d}_{p q}:\{p, q\} \neq\{i, j\},\{k, l\}\right)$ for all choices of $i, j, k, l$ distinct, where $\boldsymbol{d}_{p q}$ is the column of the matrix $D$ of Example 2.2 indexed by $\{p, q\}$. This cone thus contains the vector $\alpha=(2,2,2,2,2)$ in its relative interior. The Cox ring of $X_{\Delta}, S=\mathbb{k}\left[x_{i j}: 1 \leq i<j \leq 5\right]$, has an action of the symmetric group $S_{5}$ permuting the variables, and $S^{\alpha}$ is generated in degree one by monomials $x_{12}^{2} x_{34} x_{35} x_{45}$ and $x_{12} x_{23} x_{34} x_{45} x_{15}$ and their $S_{5}$-orbits, which are of size 10 and 12 respectively. Thus this gives an embedding of $\bar{M}_{0,5}$ into $\mathbb{P}^{21}$. The ideal $I^{\alpha}$ is then cut out by linear equations induced from multiples of the Plücker relations such as $x_{15} x_{12} x_{34} x_{23} x_{45}-x_{15} x_{12} x_{34} x_{24} x_{35}+x_{15} x_{12} x_{34}^{2} x_{25}$ and its $S_{5}$-orbit. Thus $\bar{M}_{0,5}$ is cut out as a subscheme of $\mathbb{P}^{21}$ by these linear relations and the binomial relations coming from the kernel of the surjective map $\mathbb{k}\left[z_{0}, \ldots, z_{21}\right] \rightarrow S^{\alpha}$.

We now show that other GIT quotients of $X$ can be obtained from $X / /_{n}^{\star} T^{d}$ by variation of the GIT quotient (VGIT). We assume that $\Sigma$ contains all the rays of $\Sigma^{\star}$ corresponding to rays of the Gale dual matrix $D$ (see Section 2A), and also that $D$ has no repeated columns. This means that we can write $R=\left(D \mid D C^{T}\right)=D\left(I \mid C^{T}\right)$ for some $s \times(m+1)$ matrix $C$. Let $S=\operatorname{Cox}\left(X_{\Sigma}\right)=\mathbb{k}\left[x_{0}, \ldots, x_{m}, y_{1}, \ldots, y_{s}\right]$, where $s=r-(m+1)=l-(d+1)$ and $\tilde{S}=\mathbb{k}\left[x_{0}, \ldots, x_{m}\right]$. Grade $\tilde{S}$ by deg $x_{i}=\boldsymbol{a}_{i}$, 
where $\boldsymbol{a}_{i}$ is the $i$-th column of the matrix $A$. Our assumption on $\Sigma$ means that the grading matrix for $S$ can be written in block form as

$$
\left(\begin{array}{r|r}
A & 0 \\
\hline-C & I_{S}
\end{array}\right)
$$

We write $\mathbb{N} A$ for the subsemigroup of $\mathbb{Z}^{d+1}$ generated by the columns of the matrix $A$. Let $\pi: \mathbb{Z}^{l} \rightarrow \mathbb{Z}^{d+1}$ be the projection onto the first $d+1$ coordinates, and let $\pi_{2}: \mathbb{Z}^{l} \rightarrow \mathbb{Z}^{s}$ be the projection onto the last $s=l-(d+1)$ coordinates. Recall the homomorphism $v: \mathbb{k}\left[x_{0}, \ldots, x_{m}\right] \rightarrow \mathbb{k}\left[x_{0}, \ldots, x_{m}, y_{1}^{ \pm 1}, \ldots, y_{s}^{ \pm 1}\right]$ given in the statement of Theorem 3.2. Since $D$ does not have repeated columns this is given by $v\left(x^{u}\right)=x^{u} y^{C u}$. Again $Y=Z(I)$ is the subscheme of $\mathbb{A}^{m+1+s}$ defined by the ideal $I$ of $X / /_{n}^{\star} T^{d}$.

Theorem 4.6. With the notation given above, fix $\beta \in \mathbb{N} A$. Then if $\alpha \in \mathbb{Z}^{l}$ satisfies $\pi(\alpha)=\beta$, and $\alpha_{i} \geq-\min \left\{(C u)_{i}: A u=\beta, u \in \mathbb{Q}_{\geq 0}^{m+1}\right\}$ for $1 \leq i \leq s$, then

$$
Y / / \alpha H \cong X / / \pi(\alpha) T^{d} \text {. }
$$

Proof. We will show that the map $v$ described above induces an isomorphism between $\tilde{S}^{\beta} / I(X)^{\beta}$ and $S^{\alpha} / I^{\alpha}$.

The map $v$ sends a monomial $x^{u}$ to $x^{u} y^{C u}$. Define $v^{\prime}: \tilde{S}^{\beta} \rightarrow S^{\alpha}$ by setting $v^{\prime}\left(x^{u}\right)=x^{u} y^{l \pi_{2}(\alpha)+C u}$, when $\operatorname{deg} x^{u}=l \beta$. By construction if $\operatorname{deg} x^{u}=l \beta$ then $\operatorname{deg} v^{\prime}\left(x^{u}\right)=l \alpha$, and the assumption on $\alpha$ implies that $l \pi_{2}(\alpha)+C u \in \mathbb{N}^{l-d-1}$, so the map is well-defined. Note also that $v^{\prime}$ is injective and surjective, as if $x^{u} y^{v} \in S_{l \alpha}$, then $\operatorname{deg} x^{u}=l \beta$, and $v$ must equal $C u$. We denote by $\bar{v}^{\prime}$ the induced map from $\tilde{S}^{\beta}$ to $S^{\alpha} / I^{\alpha}$, and let $J=\operatorname{ker} \bar{v}^{\prime}$. Since $\bar{v}^{\prime}$ is surjective as $v^{\prime}$ is, it remains to show that $J=I(X)^{\beta}$. Since $v^{\prime}$ is a graded homomorphism, $J$ is a homogeneous ideal, so it suffices to check that each homogeneous polynomial in $J$ lies in $I(X)$ and vice versa. Recall from Theorem 3.2 that $I=\left(v(I(X)) S_{y}\right) \cap S$, where $y=\prod_{i=0}^{m} x_{i} \prod_{j=1}^{s} y_{j}$. If $f=\sum_{u} c_{u} x^{u} \in(I(X))_{l \beta}$, then $v(f)=\sum_{u} c_{u} x^{u} y^{C u}$, so $v^{\prime}(f)=\sum c_{u} x^{u} y^{l \pi_{2}(\alpha)+C u}=y^{l \pi_{2}(\alpha)} v(f) \in I_{l \alpha}$, and thus $I(X)^{\beta} \subseteq J$.

Suppose now that $f \in J_{l \beta}$, so $v^{\prime}(f) \in I_{l \alpha}$, and thus $v^{\prime}(f)=\sum_{i} h_{i} v\left(g_{i}\right)$ for $g_{i} \in I(X)$ and $h_{i} \in S_{y}$, where we may assume that the $h_{i}$ are constant multiples of Laurent monomials, and the $g_{i}$ are homogeneous. Write $f=\sum_{u} c_{u} x^{u}, g_{i}=$ $\sum_{v} d_{v, i} x^{v}$, and $h_{i}=c_{i} x^{u_{i}^{+}} / x^{u_{i}^{-}} y^{v_{i}}$, where $u_{i}^{+}, u_{i}^{-} \in \mathbb{N}^{m+1}$, and $v_{i} \in \mathbb{Z}^{s}$. Note that $v\left(c_{i} x^{u_{i}^{+}} g_{i}\right)=c_{i} x^{u_{i}^{+}} y^{C u_{i}^{+}} v\left(g_{i}\right)$, so by changing $v_{i}$ and $g_{i}$ we may assume that $c_{i}=1$ and $u_{i}^{+}=0$ for all $i$. Also, note that the map $v$ lifts to a map from $\mathbb{k}\left[x_{0}^{ \pm 1}, \ldots, x_{m}^{ \pm 1}\right]$ to $\mathbb{k}\left[x_{0}^{ \pm 1}, \ldots, x_{m}^{ \pm 1}, y_{1}^{ \pm 1}, \ldots, y_{s}^{ \pm 1}\right]$, and $I^{\prime}(X):=I(X) \mathbb{k}\left[x_{0}^{ \pm 1}, \ldots, x_{m}^{ \pm 1}\right] \cap \tilde{S}=I(X)$ (as no irreducible component of $X$ is contained in a coordinate subspace). Also $v\left(I^{\prime}(X)\right) S_{y}=v(I(X)) S_{y}$, so we may also assume that $u_{i}^{-}=0$ for all $i$. Thus we have $v^{\prime}(f)=\sum_{i} y^{v_{i}} v\left(g_{i}\right)$ where $v_{i} \in \mathbb{Z}^{s}$, and $g_{i} \in I^{\prime}(X)_{l \beta}$ with $\tilde{g}=\sum_{i} g_{i} \in \tilde{S}$ 
because $\tilde{g}=\left.v^{\prime}(f)\right|_{y_{i}=1}$ and thus $\tilde{g} \in(I(X))_{\beta}$. Since $\operatorname{deg} v^{\prime}(f)=l \alpha$, and each $g_{i}$ is homogeneous, we have $\operatorname{deg}\left(y^{v_{i}} v\left(g_{i}\right)\right)=l \alpha$, so $v^{\prime}\left(g_{i}\right)=y^{v_{i}} v\left(g_{i}\right)$, and thus $v^{\prime}(\tilde{g})=v^{\prime}(f)$. From this it follows that $\tilde{g}=f$, since $v^{\prime}$ is injective by construction, and thus $f \in I(X)_{l \beta}$, so $J=I(X)^{\beta}$ as required.

Corollary 4.7. The Chow and Hilbert quotients $X /{ }^{\mathrm{Ch}} T^{d}$ and $X /{ }^{\mathrm{H}} T^{d}$ and the GIT quotients $X / / \alpha T^{d}$ are related by variation of the GIT quotient.

Proof. It remains to check that an $\alpha$ satisfying the hypotheses of Theorem 4.6 actually exists. This means checking that the set $\left\{(C u)_{i}: A u=\beta, u \in \mathbb{Q}_{\geq 0}^{m+1}\right\}$ is bounded below so the minimum exists. This follows from the fact that $\{u: \bar{A} u=\beta$, $\left.u \in \mathbb{Q}_{\geq 0}^{m+1}\right\}$ is a polytope, since the all-ones vector is in the row space of $A$. Thus the lower bound is the minimum of a linear functional on a polytope, which is finite.

\section{A toric variety containing $\bar{M}_{0, n}$}

In the remainder of the paper we apply the previous theorems to obtain equations for $\bar{M}_{0, n}$. Using the construction given in Example 3.1, $\bar{M}_{0, n}$ is a subvariety of the normalization of the Chow or Hilbert quotient of $\mathbb{P}^{\left(\begin{array}{c}n \\ 2\end{array}\right)-1}$ by $T^{n-1}$. In this section we describe a smooth normal toric variety $X_{\Delta}$ that is a sufficiently large toric subvariety of both $X_{\Sigma^{\mathrm{Ch}}}$ and $X_{\Sigma^{\mathrm{H}}}$. We prove in Theorem 5.7 that $\bar{M}_{0, n}$ is contained in $X_{\Delta}$.

We first define a simplicial complex and set of vectors that form the underlying combinatorics of $\Delta$. Recall that $[n]=\{1, \ldots, n\}$, and for $I \subset[n]$ we have $I^{c}=[n] \backslash I$.

Definition 5.1. Let $\mathscr{I}=\left\{I \subset[n]: 1 \in I,|I| \geq 2,\left|I^{c}\right| \geq 2\right\}$. The simplicial complex $\tilde{\Delta}$ has vertices $\mathscr{I}$ and $\sigma \subseteq \mathscr{I}$ is a simplex of $\tilde{\Delta}$ if for all $I$ and $J \in \sigma$ we have $I \subseteq J$, $J \subseteq I$, or $I \cup J=[n]$.

Let $\mathscr{\mathscr { C }}=\{i j: 2 \leq i<j \leq n, i j \neq 23\}$ be an indexing set for a basis of $\mathbb{R}^{\left(\begin{array}{l}n \\ 2\end{array}\right)-n}$. For each $I \in \mathscr{I}$, define the vector

$$
\boldsymbol{r}_{I}=\left(R_{i j, I}\right)_{i j \in \mathscr{E}} \in \mathbb{Z}^{\left(\begin{array}{c}
n \\
2
\end{array}\right)-n}, \quad R_{i j, I}=\left\{\begin{aligned}
1 & \text { if }|I \cap\{i j\}|=0,|I \cap\{23\}|>0, \\
-1 & \text { if }|I \cap\{i j\}|>0,|I \cap\{23\}|=0, \\
0 & \text { otherwise. }
\end{aligned}\right.
$$

Form the $\left(\left(\begin{array}{l}n \\ 2\end{array}\right)-n\right) \times|\mathscr{I}|$ matrix $R$ with $I$-th column $\boldsymbol{r}_{I}$.

Note that the set $\mathscr{I}$ also labels the boundary divisors $\delta_{I}$ of $\bar{M}_{0, n}$, and a simplex $\sigma$ lies in $\tilde{\Delta}$ precisely if the corresponding boundary divisors intersect nontrivially. Proposition 5.2. The collection of cones $\left\{\operatorname{pos}\left(\boldsymbol{r}_{I}: I \in \sigma\right): \sigma \in \tilde{\Delta}\right\}$ is a polyhedral fan $\Delta$ in $\mathbb{R}^{\left(\begin{array}{c}n \\ 2\end{array}\right)-n}$ of dimension $n-3$. The associated toric variety $X_{\Delta}$ is smooth, and the support of $\Delta$ is the tropical variety of $M_{0, n} \subset T^{\left(\begin{array}{l}n \\ 2\end{array}\right)-n}$. 
This fan is well known in the literature as the space of phylogenetic trees [Speyer and Sturmfels 2004, Section 4, Buneman 1974; Vogtmann 1990; Billera et al. 2001]. To prove Proposition 5.2 and for results in the remainder of this section, we use the following notation.

Definition 5.3. Fix $n \geq 6$. Let $A_{n}$ be the $n \times\left(\begin{array}{l}n \\ 2\end{array}\right)$-dimensional matrix with $i j$-th column equal to $e_{i}+e_{j}$ and let $D$ be the $\left(\left(\begin{array}{l}n \\ 2\end{array}\right)-n\right) \times\left(\begin{array}{l}n \\ 2\end{array}\right)$ matrix

$D=\left(D_{i j, k l}\right)_{\substack{i j \in \mathscr{E} \\ 1 \leq k<l \leq n}}, \quad D_{i j, k l}=\left\{\begin{aligned} 1 & \text { if } k l=i j, \text { or } k l \in\{12,13\},|\{i j\} \cap\{k l\}|=\varnothing, \\ -1 & \text { if } k l=23, \text { or } k l \in\{1 i, 1 j\},|\{23\} \cap\{k l\}|=\varnothing, \\ 0 & \text { otherwise. }\end{aligned}\right.$

Let $C$ be the $\left(|\mathscr{I}|-\left(\begin{array}{l}n \\ 2\end{array}\right)\right) \times\left(\begin{array}{l}n \\ 2\end{array}\right)$ matrix with rows indexed by $I \in \mathscr{I}$ with $3 \leq|I| \leq n-3$, columns indexed by $\{i j: 1 \leq i<j \leq n\}$, and entries

$$
C_{I, i j}= \begin{cases}1 & \text { if } i, j \in I, \\ 0 & \text { otherwise. }\end{cases}
$$

A straightforward calculation from the definition of $D$ shows that

$$
R=D\left(I \mid C^{T}\right) .
$$

The matrix $A_{n}$ is the vertex-edge incidence matrix for the complete graph on $n$ vertices and $D$ is its Gale dual, so $D A_{n}^{T}=0$. Note that the square $\left(\left(\begin{array}{l}n \\ 2\end{array}\right)-n\right) \times$ $\left(\left(\begin{array}{l}n \\ 2\end{array}\right)-n\right)$ submatrix of $D$ with columns indexed by $\mathscr{E}$ is the identity matrix. In particular, $D$ has rank $\left(\begin{array}{l}n \\ 2\end{array}\right)-n$.

Proof of Proposition 5.2. Let the lattice $L$ be the integer row space of $A_{n}$. The affine cone over the Grassmannian $G(2, n)$ in its Plücker embedding is a subvariety

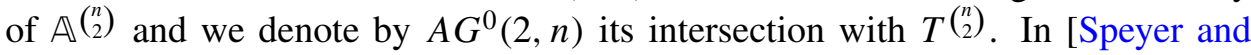
Sturmfels 2004] it is shown that the tropical variety of $A G^{0}(2, n)$ is a $(2 n-3)$ dimensional fan in $\mathbb{R}^{\left(\begin{array}{c}n \\ 2\end{array}\right)}$ with lineality space $L \otimes \mathbb{R}$, and the simplicial complex corresponding to the fan structure on $\operatorname{Trop}\left(A G^{0}(2, n)\right)$ is $\tilde{\Delta}$. Specifically, the cone corresponding to the cone $\sigma \in \tilde{\Delta}$ is $\operatorname{pos}\left(\boldsymbol{e}_{I}: I \in \sigma\right)+L$, where

$$
\boldsymbol{e}_{I}=\sum_{i \in I, j \notin I} \boldsymbol{e}_{i j} \in \mathbb{Z}^{\left(\begin{array}{c}
n \\
2
\end{array}\right)} \text {. }
$$

Recall from Example 3.1 that $M_{0, n}=G^{0}(2, n) / T^{n-1}$. Using Proposition 2.1, one can show that for any $X \subseteq T^{m}$ that is invariant under the action of a torus $T^{d} \subset T^{m}$, the tropical variety of $X / T^{d} \subset T^{m} / T^{d}$ is equal to the quotient of the tropical variety of $X \subset T^{m}$ by the tropical variety of $T^{d}$. Thus $\operatorname{Trop}\left(M_{0, n}\right)=$ $\operatorname{Trop}\left(G^{0}(2, n)\right) / \operatorname{Trop}\left(T^{n-1}\right)=\operatorname{Trop}\left(A G^{0}(2, n)\right) / L$. To prove the first part of the

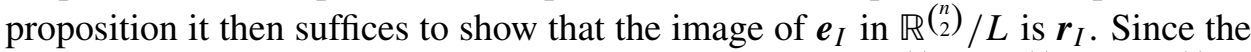

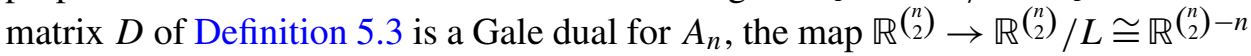


given by sending the basis vector $\boldsymbol{e}_{i j}$ of $\mathbb{R}^{\left(\begin{array}{l}n \\ 2\end{array}\right)}$ to $-\frac{1}{2} \boldsymbol{d}_{i j}$ is an isomorphism, where $\boldsymbol{d}_{i j}$ is the $i j$-th column of $D$. The image of $\boldsymbol{e}_{I}$ under this map is then $-\frac{1}{2} \sum_{i \in I, j \notin I} \boldsymbol{d}_{i j}$. Since $\sum_{1 \leq i<j \leq n} \boldsymbol{d}_{i j}=\mathbf{0}$, this is $\frac{1}{2} \sum_{i, j \in I} \boldsymbol{d}_{i j}+\frac{1}{2} \sum_{i, j \in I^{c}} \boldsymbol{d}_{i j}$. Let $l_{i}$ denote the $i$-th row of the matrix $A_{n}$, and note that $\sum_{i \in I} l_{i}-\sum_{i \notin I} l_{i}=\sum_{i, j \in I} \boldsymbol{e}_{i j}-\sum_{i, j \in I^{c}} \boldsymbol{e}_{i j}$, so $\sum_{i, j \in I} \boldsymbol{d}_{i j}=\sum_{i, j \in I^{c}} \boldsymbol{d}_{i j}$. Thus the image of $\boldsymbol{e}_{I}=\sum_{i, j \in I} \boldsymbol{d}_{i j}=\sum_{i, j \in I^{c}} \boldsymbol{d}_{i j}$. Since this is equal to $\left(D\left(I \mid C^{T}\right)\right)_{I}=\boldsymbol{r}_{I}$, we conclude that $\boldsymbol{r}_{I}$ is the image of $\boldsymbol{e}_{I}$. Note that this map is induced from a map of lattices, as the relevant lattice in $\mathbb{R}\left(\begin{array}{c}n \\ 2\end{array}\right)$ is the index two sublattice of $\mathbb{Z}\left(\begin{array}{l}n \\ 2\end{array}\right)$ with even coordinate sum.

The fact that $\Delta$ is simplicial of dimension $n-3$ is due to [Robinson and Whitehouse 1996]. Recall that a fan is smooth if for each cone the intersection of the lattice with the linear span of that cone is generated by the first lattice points on each ray of the cone. The fact that the fan is smooth follows from the work of Feichtner and Yuzvinsky. In [Feichtner 2006] it is shown that the fan $\Delta$ is the one associated to the nested set complex for a related hyperplane arrangement, while in [Feichtner and Yuzvinsky 2004, Proposition 2] it is shown that the fans associated to nested set complexes are smooth.

Notation 5.4. Let $A_{n}$ and $D$ be the matrices described in Definition 5.3. Recall that the chamber complex $\Sigma(D)$ is the polyhedral fan in $\mathbb{R}_{\left(\begin{array}{l}n \\ 2\end{array}\right)-n}$ subdividing the cone spanned by the columns of $D$ obtained by intersecting all simplicial cones spanned by columns of $D$. This is equal to the secondary fan of $A_{n}$, and thus to the fan of the toric variety $X_{\Sigma^{\mathrm{Ch}}}$ (see [Billera et al. 1990]). Recall also that the regular subdivision $\Delta_{w}$ of the configuration of the columns $\boldsymbol{a}_{i j}$ of $A_{n}$ corresponding to a vector $w \in \mathbb{R}^{\left(\begin{array}{l}n \\ 2\end{array}\right)}$ has $\operatorname{pos}\left(\boldsymbol{a}_{i j}: i j \in \sigma\right)$ as a cell for $\sigma \subseteq\{i j: 1 \leq i<j \leq n\}$ if and only if there is some $\boldsymbol{c} \in \mathbb{R}^{n}$ such that $\boldsymbol{c} \cdot \boldsymbol{a}_{i j}=w_{i j}$ for $i j \in \sigma$, and $\boldsymbol{c} \cdot \boldsymbol{a}_{i j}<w_{i j}$ for $i j \notin \sigma$ (see [Gel'fand et al. 1994; Sturmfels 1996; Maclagan and Thomas 2007] for background on regular subdivisions). We denote by $\mathbb{N} A_{n}$ the subsemigroup of $\mathbb{N}^{n}$ generated by the columns of $A_{n}$, and by $\mathbb{R}_{\geq 0} A_{n}$ the cone in $\mathbb{R}^{n}$ whose rays are the positive spans of the columns of $A_{n}$.

Proposition 5.5. The fan $\Delta$ is a subfan of the fan $X_{\Sigma^{\mathrm{Ch}}}$, so $X_{\Delta}$ is a toric subvariety of $X_{\Sigma^{\mathrm{ch}}}$.

Proof. Let $\sigma$ be a top-dimensional cone of $\Delta$. Then $\sigma=\operatorname{pos}\left(\boldsymbol{r}_{I_{1}}, \ldots, \boldsymbol{r}_{I_{n-3}}\right)$, where $I_{i} \subseteq I_{j}, I_{j} \subseteq I_{i}$, or $I_{i} \cup I_{j}=[n]$. There is a trivalent (phylogenetic) tree $\tau$ with $n$ labeled leaves such that the $I_{j}$ correspond to the splits obtained by deleting internal edges of $\tau$.

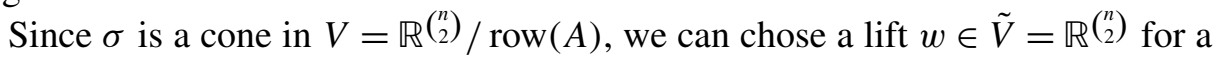
vector in $\sigma$, and thus consider the regular subdivision $\Delta_{w}$ of the configuration $A_{n}$, which does not depend on the choice of lift.

To show that $\sigma$ is a cone in the chamber complex of $D$ we first characterize the subdivision $\Delta_{w}$ coming from a lift $w$ of a vector in $\sigma$. To an internal vertex $v$ of 
the phylogenetic tree $\tau$ we associate the set $\mathscr{C}_{v}$ of pairs $i j$ such that the path in $\tau$ between the vertices labeled $i$ and $j$ passes through $v$, and the cone $C_{v}=\operatorname{pos}\left(\boldsymbol{e}_{i j}\right.$ : $\left.i j \in \mathscr{C}_{v}\right) \subseteq \mathbb{R}^{n}$. The cone $C_{v}$ is obtained by taking the cone over the polytopes $M\left(\cong_{v}\right)$ of [Kapranov 1993, Remark 1.3.7].

We claim that for any lift $w \in \mathbb{R}^{\left(\begin{array}{l}n \\ 2\end{array}\right)}$ of a vector in $\sigma$ the subdivision $\Delta_{w}$ has cones $C_{v}$ for $v$ an internal vertex of $\tau$. To see this, for each $\boldsymbol{r}_{I} \in \sigma$ set $I^{\prime}=[n] \backslash I$ if the path from leaf $i$ to leaf $j$ in $\tau$ passes through $v$ for some $i, j \in I$, and $I^{\prime}=I$ otherwise. This ensures that $i j \notin \mathscr{C}_{v}$ for $i, j \in I^{\prime}$. If $\sum_{i=1}^{n-3} a_{i} \boldsymbol{r}_{I_{i}}$ is a point in $\sigma$ with $a_{i}>0$ for all $i$, we can choose the lift $w=\sum_{i=1}^{n-3} a_{i} \sum_{i, j \in I_{i}^{\prime}} \boldsymbol{e}_{i j}$. Since $w_{i j}>0$ for $i j \notin \mathscr{C}_{v}$, and $w_{i j}=0$ for $i j \in \mathscr{C}_{v}$, taking $\boldsymbol{c}=\mathbf{0}$ we see that $C_{v}$ is a face of $\Delta_{w}$.

This shows that the given collection are cones in the subdivision $\Delta_{w}$. That they cover all of the cone generated by the $\boldsymbol{a}_{i j}$ is [Kapranov 1993, Claim 1.3.9]. For the reader's convenience we give a self-contained proof. To show that we are not missing anything, it suffices to show that any $\boldsymbol{v} \in \mathbb{N} A_{n}$ lies in $C_{v}$ for some $C_{v}$, which will show that the $C_{v}$ cover $\mathbb{R}_{\geq 0} A_{n}$. If $\boldsymbol{v}$ lies in $\mathbb{N} A_{n}$ then there is a graph $\Gamma$ on $n$ vertices with degree sequence $\boldsymbol{v}$. We may assume that $\Gamma$ has the largest edge sum out of all graphs with degree sequence $\boldsymbol{v}$, where an edge $i j$ has weight the number of internal edges in the path between $i$ and $j$ in the tree $\tau$. This means that if $i j$ and $k l$ are two edges of $\Gamma$ with $|\{i, j, k, l\}|=4$, the paths in $\tau$ corresponding to these two edges must cross, as otherwise we could get a larger weight by replacing these two edges by the pair with the same endpoints that do cross. We claim that there is then some vertex $v \in \tau$ for which the path in $\tau$ corresponding to each edge in $\Gamma$ passes through $v$, which will show that $v \in C_{v}$. The statement is trivial if $\Gamma$ has at most two edges, since any two paths must intersect. The set of collections of edges of $\Gamma$ for which the corresponding paths share a common vertex forms a simplicial complex, so if the claim is false, we can find a subgraph $\Gamma^{\prime}$ of $\Gamma$ for which there is no vertex of $\tau$ through which all of the corresponding paths pass, but every proper subgraph of $\Gamma^{\prime}$ has the desired property $\left(\Gamma^{\prime}\right.$ is a minimal nonface of the simplicial complex). The subgraph $\Gamma^{\prime}$ must have at least three edges. Pick three edges $e_{1}, e_{2}, e_{3}$ of $\Gamma^{\prime}$, and let $v_{i}$ for $i \in\{1,2,3\}$ be a vertex of $\tau$ for which the path corresponding to each vertex of $\Gamma^{\prime}$ except $e_{i}$ passes. Since $\tau$ is a tree one of these vertices lies in the path between the other two; without loss of generality we assume that $v_{2}$ lies between $v_{1}$ and $v_{3}$. But the path corresponding to $e_{2}$ passes through $v_{1}$ and $v_{3}$ while avoiding $v_{2}$, a contradiction.

This shows that the subdivision corresponding to a vector in the interior of $\sigma$ is the same subdivision $\Delta_{\sigma}$ for any vector in $\sigma$, and that $\sigma$ lies inside the cone $\bigcap_{v} \operatorname{pos}\left(\boldsymbol{r}_{i j}: i j \notin \mathscr{C}_{v}\right)$ of the chamber complex of $D$. To finish the proof, we show that this cone lies in $\sigma$. The cone $\sigma$ has the following facet description: for each quadruple $i, j, k, l$ two of the pairs of paths $\{i j, k l\},\{i k, j l\}$, and $\{i l, j k\}$ in the tree $\tau$ have the same combined length, and one pair is shorter. Without loss 
of generality we assume that $i j, k l$ is the shorter pair. This gives the inequality $w_{i j}+w_{k l} \geq w_{i k}+w_{j l}=w_{i l}+w_{j k}$. The set of these inequalities as $\{i, j, k, l\}$

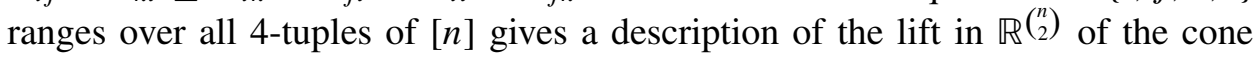
$\sigma$ (see [Speyer and Sturmfels 2004, Theorem 4.2]). Note that row $(A)$ lies in this cone, so to show that a vector $v$ in the relative interior of $\bigcap_{v} \operatorname{pos}\left(\boldsymbol{r}_{i j}: i j \notin \mathscr{C}_{v}\right)$ lies in $\sigma$, it suffices to show that for each inequality there is some lift of $\boldsymbol{v}$ to $\mathbb{R}^{\left(\begin{array}{l}n \\ 2\end{array}\right)}$ that satisfies that inequality. Given such a $v$, and a 4-tuple $\{i, j, k, l\}$ giving the inequality $w_{i j}+w_{k l} \geq w_{i k}+w_{j l}=w_{i l}+w_{j k}$, pick a vertex $v$ in $\tau$ that lies in all of the paths $i k, j l, i l$, and $j k$. Then since $v \in \operatorname{relint}\left(\operatorname{pos}\left(\boldsymbol{r}_{i j}: i j \notin \mathscr{C}_{v}\right)\right)$, there is a lift $w$ of $\boldsymbol{v}$ with $w_{i k}=w_{j l}=w_{i l}=w_{j k}=0$, and $w_{i j}, w_{k l} \geq 0$, which satisfies the given inequality. We conclude that $\bigcap_{v} \operatorname{pos}\left(\boldsymbol{r}_{i j}: i j \notin \mathscr{C}_{v}\right) \subseteq \sigma$, and thus we have equality, so $\sigma$ is a cone in the chamber complex of $D$.

Recall that an open cone $\sigma \in \Sigma^{\mathrm{H}}$ consists of $w$ for which the saturation of $\operatorname{in}_{w}\left(I_{A}\right)$ is constant, where we here follow the standard convention that the leading form $\operatorname{in}_{w}(f)$ of a polynomial $f$ consists of terms of largest $w$-degree.

Proposition 5.6. The fan $\Delta$ is a subfan of the fan $\Sigma^{\mathrm{H}}$, so $X_{\Delta}$ is a toric subvariety of $X_{\Sigma^{\mathrm{H}}}$.

Proof. Continuing with the notation from the proof of Proposition 5.5, we first show that $\operatorname{in}_{w}\left(I_{A_{n}}\right)$ is constant for all lifts $w$ of vectors in a maximal cone $\sigma$ of $\Delta$ corresponding to a phylogenetic tree $\tau$. A planar representation of $\tau$ with the vertices on a circle determines a circular order on $[n]$. Without loss of generality we may assume that this is the standard increasing order. Draw the complete graph $K_{n}$ on the circle with the same order. By [Sturmfels 1996, Theorem 9.1] a reduced Gröbner basis $\mathscr{B}$ for $I_{A_{n}}$ is given by binomials of the form $x_{i j} x_{k l}-x_{i k} x_{j l}$, where the edges $i k$ and $j l$ of $K_{n}$ cross but the edges $i j$ and $k l$ do not. The open Gröbner

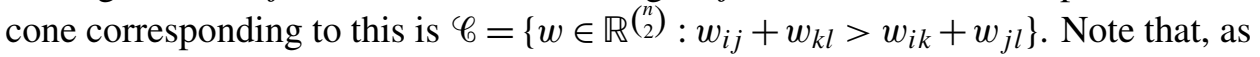
in the proof of Proposition 5.5, the lift of any vector in $\sigma$ lies on the boundary of $\mathscr{C}$, and so there is a term order $\prec$ for which the initial ideal $\operatorname{in}_{\prec}\left(\operatorname{in}_{w}\left(I_{A_{n}}\right)\right)$ equals the ideal $\left\langle x_{i j} x_{k l}: i j, k l\right.$ do not cross in $\left.K_{n}\right\rangle$, where $w$ is a lift of any vector in $\sigma$. Thus by [Sturmfels 1996, Corollary 1.9], a Gröbner basis for $I_{A_{n}}$ with respect to the weight order given by such a $w$ is obtained by taking the initial terms with respect to $w$ of the Gröbner basis $\mathscr{B}$. For a binomial $x_{i j} x_{k l}-x_{i k} x_{j l}$, where the edges $i k$ and $j l$ of $K_{n}$ cross but the edges $i j$ and $k l$ do not, either the paths $i k$ and $j l$ also cross in $\tau$, or they do not. If the paths do cross, the lift $w$ of a vector in $\sigma$ has $w_{i k}+w_{j l}=w_{i j}+w_{j l}$, and if they do not there is an internal edge of $\tau$ in the paths $i k$ and $j l$ but not the paths $i j$ and $k l$, so if $w$ lies in the interior of $\sigma$ we have $w_{i k}+w_{j l}<w_{i j}+w_{j l}$. This means that the initial ideal is determined by the tree $\tau$, so $\operatorname{in}_{w}\left(I_{A_{n}}\right)$ is constant for all lifts $w$ of vectors in $\sigma$. This shows that $\sigma$ is contained in a cone $\sigma^{\prime}$ of $\Sigma^{\mathrm{H}}$. 
It remains to show that $\sigma=\sigma^{\prime}$. This follows from Proposition 5.5, which shows that $\sigma$ is a cone in the secondary fan of $I_{A_{n}}$, since the Gröbner fan refines the secondary fan [Sturmfels 1996, Proposition 8.15]. The fan $\Sigma^{\mathrm{H}}$ is obtained from the Gröbner fan of $I_{A_{n}}$ by amalgamating cones corresponding to initial ideals with the same saturation with respect to $\left\langle x_{i j}: 1 \leq i<j \leq n\right\rangle$, so the result follows, since ideals with the same saturation have the same radical and thus the cones live in the same secondary cone.

We are now able to show that $\bar{M}_{0, n}$ is a subvariety of $X_{\Delta}$. As described in Example 3.1 the moduli space $\bar{M}_{0, n}$ is both the Chow and Hilbert quotient of the Grassmannian $G(2, n)$ by the torus $T^{n-1}$.

Theorem 5.7. The toric variety $X_{\Delta}$ is the union of those $T^{\left(\begin{array}{c}n \\ 2\end{array}\right)-n}$-orbits of $X_{\Sigma^{\star}}$ intersecting the closure of $M_{0, n}$ in this $X_{\Sigma^{\star}}$. The closure of $M_{0, n} \subseteq T^{\left(\begin{array}{l}n \\ 2\end{array}\right)-n}$ inside $X_{\Delta}$ is equal to $\bar{M}_{0, n}$.

We remark that the second part of this result was originally observed by related methods in [Tevelev 2007, Theorem 5.5].

Proof of Theorem 5.7. By Propositions 5.5 and 5.6 we know that $\Delta$ is a subfan of both $\Sigma^{\mathrm{Ch}}$ and $\Sigma^{\mathrm{H}}$, and by Proposition 5.2 we know that the support of $\Delta$ is the tropical variety of $M_{0, n} \subset T^{\left(\begin{array}{l}n \\ 2\end{array}\right)-n}$. Corollary 2.7 says that the closure of $M_{0, n} \subset$ $T^{\left(\begin{array}{c}n \\ 2\end{array}\right)-n}$ inside $X_{\Sigma^{\star}}$ intersects the orbit corresponding to a cone $\sigma \in \Sigma^{\star}$ if and only the tropical variety of $M_{0, n}$ intersects the interior of $\sigma$. Thus the orbits of $X_{\Sigma^{\star}}$ intersecting this closure are precisely the orbits in the toric subvariety $X_{\Delta}$. This proves the first assertion of the theorem. For the second, note that the closure of $M_{0, n}$ in $X_{\Delta}$ is $G(2, n) /{ }_{n}^{\star} T^{n-1}$, and since $\bar{M}_{0, n}=G(2, n) / /^{\star} T^{n-1}$ is smooth and irreducible, this is equal to $\bar{M}_{0, n}$ by Theorem 3.2.

Remark 5.8. We emphasize that although we know that the fans $\Sigma^{\mathrm{Ch}}$ and $\Sigma^{\mathrm{H}}$ are the secondary and saturated Gröbner fans respectively, we do not even know how many rays each has. So while in theory one could describe equations for $\bar{M}_{0, n}$ in the Cox rings of these toric varieties, this is not possible in practice. On the other hand, $\Delta$ has a completely explicit description for all $n$. In particular, this makes the Cox ring of $X_{\Delta}$ accessible and enables us to derive equations for $\bar{M}_{0, n}$ inside it.

\section{Equations for $\bar{M}_{0, n}$}

In this section we apply Theorem 3.2 to give equations for $\bar{M}_{0, n}$ in the Cox ring of the toric variety $X_{\Delta}$ described in Section 5 .

Recall that $\mathscr{I}=\left\{I \subset[n]: 1 \in I,|I|,\left|I^{c}\right| \geq 2\right\}$, and there is a ray $\operatorname{pos}\left(\boldsymbol{r}_{I}\right)$ of $\Delta$ for each $I \in \mathscr{I}$. The Cox ring of $X_{\Delta}$ is $S=\mathbb{k}\left[x_{I}: I \in \mathscr{I}\right]$, with $\operatorname{deg} x_{I}=\left[D_{I}\right] \in \mathrm{Cl}\left(X_{\Delta}\right)$, where $D_{I}$ is the torus-invariant divisor corresponding to the ray through $\boldsymbol{r}_{I}$. We 
construct the grading matrix as follows. Set $b=|\mathscr{I}|-\left(\begin{array}{l}n \\ 2\end{array}\right)$ for $n \geq 5$. Let $G$ be the $(n+b) \times|\mathscr{I}|$ matrix that is given in block form by

$$
G=\left(\begin{array}{c|c}
A_{n} & 0 \\
\hline-C & I_{b}
\end{array}\right)
$$

where 0 denotes the $n \times b$ zero matrix, $A_{n}$ and $C$ are as in Definition 5.3, and $I_{b}$ is the $b \times b$ identity matrix. For $n=4$ we set $G=\left(\begin{array}{lll}1 & 1 & 1\end{array}\right)$.

As the following lemma shows, this form of $G$ is consistent with the choice of grading matrix for the Cox ring of $X_{\Delta}$ suggested in (3). The lemma also shows that $\mathrm{Cl}\left(X_{\Delta}\right) \cong \operatorname{Pic}\left(\bar{M}_{0, n}\right)$, so we first recall the description of $\operatorname{Pic}\left(\bar{M}_{0, n}\right)$. For $I \subset[n]$ with $1 \notin I$ the notation $\boldsymbol{e}_{I}$ means the basis element $\boldsymbol{e}_{I^{c}}$ of $\mathbb{Z}^{|\mathscr{\Phi}|}$.

Proposition 6.1 [Keel 1992, Theorem 1]. Let $W$ be the sublattice of $\mathbb{Z}^{\left|{ }^{\mid}\right|}$spanned by the vectors

$$
w_{i j k l}=\prod_{\substack{i, j \in I \\ k, l \notin I}} \boldsymbol{e}_{I}-\prod_{\substack{i, l \in I \\ j, k \notin I}} \boldsymbol{e}_{I},
$$

where $\{i, j, k, l\} \subseteq[n]$ has size four. The Picard group of $\bar{M}_{0, n}$ is isomorphic to $\mathbb{Z}^{\mid \mathscr{}} / W$.

Lemma 6.2. For $n \geq 5$ the divisor class group of $X_{\Delta}$ is isomorphic to $\mathbb{Z}^{b+n}$, with the image of $\left[D_{I}\right]$ under this isomorphism equal to the column $\boldsymbol{g}_{I}$ of the matrix $G$ indexed by $I \in \mathscr{I}$. We have $\mathrm{Cl}\left(X_{\Delta}\right) \cong \operatorname{Pic}\left(\bar{M}_{0, n}\right)$, with the isomorphism taking $\left[D_{I}\right]$ to the boundary divisor $\delta_{I}$.

Proof. That $\mathrm{Cl}\left(X_{\Delta}\right) \cong \mathbb{Z}^{b+n}$ follows from the short exact sequence $(\dagger)$ computing the class group of a toric variety [Fulton 1993, p. 63], together with Proposition 5.2 , since smooth toric varieties have torsion-free divisor class groups. To see that the image of $\left[D_{I}\right]$ is $g_{I}$, the $I$-th column of $G$, it suffices to show that the matrix $G$ is a Gale dual for the matrix $R$, so the exact sequence $(\dagger)$ is

$$
0 \rightarrow M \stackrel{R^{T}}{\rightarrow} \mathbb{Z}^{\mid \mathscr{}} \stackrel{G}{\rightarrow} \mathrm{Cl}\left(X_{\Delta}\right) \rightarrow 0
$$

Now $G R^{T}=G\left(I \mid C^{T}\right)^{T} D^{T}$, so

$$
G R^{T}=\left(\begin{array}{c|c}
A_{n} & 0 \\
\hline-C & I
\end{array}\right)\left(\frac{I}{C}\right) D^{T}=\left(\begin{array}{c}
A D^{T} \\
\hline 0
\end{array}\right)=0 .
$$

Finally, to show that $\mathrm{Cl}\left(X_{\Delta}\right) \cong \operatorname{Pic}\left(\bar{M}_{0, n}\right)$, since $\mathrm{Cl}\left(X_{\Delta}\right) \cong \mathbb{Z}^{\left|{ }^{\mathscr{F}}\right|} / \mathrm{im}_{\mathbb{Z}} R^{T}$ and $\operatorname{Pic}\left(\bar{M}_{0, n}\right) \cong \mathbb{Z}^{\mid \Phi^{\mid}} / W$, it suffices to show that $W=\operatorname{im}_{\mathbb{Z}} R^{T}$. Since $\operatorname{im}_{\mathbb{Z}} R^{T}=\operatorname{ker}_{\mathbb{Z}} G$, both lattices $W$ and im $R^{T}$ are saturated, and $\operatorname{rank} W=\operatorname{rank} R^{T}=\left(\begin{array}{l}n \\ 2\end{array}\right)-n$, it suffices to show that each $w_{i j k l}$ lies in the kernel of $G$. Now $w_{i j k l}$ restricted to the sets $I$ with $|I|=2$ or $|I|=n-2$ is $\boldsymbol{e}_{i j}+\boldsymbol{e}_{k l}-\boldsymbol{e}_{i l}-\boldsymbol{e}_{j k}$, which lies in ker $A_{n}$. Restricting $w_{i j k l}$ to $\left\{\boldsymbol{e}_{s t}: s, t \in I\right\} \cup\left\{\boldsymbol{e}_{I}\right\}$ we see that $\left(-C \mid I_{b}\right) w_{i j k l}=0$. For example, if 
$1, i, j, k, l \in I$ then the sum is $-1-1+1+1+0=0$. Thus $w_{i j k l} \in \operatorname{ker} G$ as required.

For $n=4$ we have $\mathrm{Cl}\left(X_{\Delta}\right) \cong \operatorname{Pic}\left(\bar{M}_{0,4}\right) \cong \mathbb{Z}$, and the image of the $\boldsymbol{g}_{i j}$ is $1 \in \mathbb{Z}$, which is also equal to each $\left[D_{i j}\right]$.

We begin by proving the first part of Theorem 1.2. Recall that

$$
\mathscr{I}=\left\{I \subset[n]: 1 \in I,|I| \geq 2,\left|I^{c}\right| \geq 2\right\} .
$$

If $1 \notin I \subseteq[n]$ then by $x_{I}$ we mean the variable $x_{[n] \backslash I}$ in the Cox ring $S=\mathbb{k}\left[x_{I}: I \in \mathscr{I}\right]$ of $X_{\Delta}$.

Theorem 6.3. For $n \geq 5$ the equations for $\bar{M}_{0, n}$ in the Cox ring of $X_{\Delta}$ are obtained by homogenizing the Plücker relations with respect to the grading of $S$ and then saturating by the product of the variables of $S$. Specifically, the ideal is

$$
I\left(\bar{M}_{0, n}\right)=\left(\left\langle\prod_{\substack{i j \in I \\ k l \notin I}}-\prod_{\substack{i k \in I \\ j l \notin I}} x_{I}+\prod_{\substack{i l \in I \\ j k \notin I}} x_{I}\right\rangle:\left(\prod_{I} x_{I}\right)^{\infty}\right),
$$

where the generating set runs over all $\{i, j, k, l\}$ with $1 \leq i<j<k<l \leq n$.

Before proving the theorem, we first find equations for the intersection $M_{0, n}$ of $\bar{M}_{0, n}$ with the torus $\mathbb{T}=T^{\left(\begin{array}{l}n \\ 2\end{array}\right)-1} / T^{n-1} \cong\left(\mathbb{k}^{\times}\right)\left(\begin{array}{c}n \\ 2\end{array}\right)-n$ of the toric variety $X_{\Delta}$. The coordinates for $\mathbb{T}$ are labeled by $\mathscr{E}=\{i j: 2 \leq i<j \leq n, i j \neq 23\}$. Recall that the ideal of $G(2, n) \subset \mathbb{P}\left(\begin{array}{l}n \\ 2\end{array}\right)-1$ is generated by the Plücker relations $p_{i j k l}=$ $x_{i j} x_{k l}-x_{i k} x_{j l}+x_{i l} x_{j k}$ :

$$
I_{2, n}=\left\langle x_{i j} x_{k l}-x_{i k} x_{j l}+x_{i l} x_{j k}: 1 \leq i<j<k<l \leq n\right\rangle .
$$

Proposition 6.4. The intersection $M_{0, n}=\left(Z\left(I_{2, n}\right) \cap T^{\left.\left(\begin{array}{l}n \\ 2\end{array}\right)\right)}\right) / T^{n} \subseteq \mathbb{T}$ is cut out by the equations

$$
J=\left\langle z_{k l}-z_{2 l}+z_{2 k}: 3 \leq k \leq l \leq n\right\rangle \subseteq \mathbb{k}\left[z_{i j}^{ \pm 1}: i j \in \mathscr{\mathscr { C }}\right],
$$

where we set $z_{23}=1$.

Proof. We first show that $\left(Z\left(I_{2, n}\right) \cap T^{\left(\begin{array}{l}n \\ 2\end{array}\right)}\right) / T^{n}$ is defined by the ideal

$$
J^{\prime}=\left\langle z_{i j} z_{k l}-z_{i k} z_{j l}+z_{i l} z_{j k}: 1 \leq i<j<k<l \leq n\right\rangle \subseteq \mathbb{k}\left[z_{i j}^{ \pm 1}: i j \in \mathscr{E}\right],
$$

where we set $z_{i j}=1$ when $i j \notin \mathscr{E}$. The content of Proposition 2.1 is that the relevant ideal is $\phi^{-1}\left(I_{2, n}\right)$, where $\phi: \mathbb{k}\left[z_{i j}^{ \pm 1}: i j \in \mathscr{E}\right] \rightarrow \mathbb{k}\left[x_{i j}^{ \pm 1}: 1 \leq i<j \leq n\right]$ is given by $\phi\left(z_{i j}\right)=\prod_{k l} x_{k l}^{D_{i j, k l}}$.

Since the map $\phi$ is an injection, to show that $J^{\prime}$ is the desired ideal we just need to show that the generators of $J^{\prime}$ are taken to a generating set for $I_{2, n} \subseteq \mathbb{k}\left[x_{i j}^{ \pm 1}\right]$ 
by $\phi$. When $i j \in \mathscr{E}$ we have

$$
\phi\left(z_{i j}\right)= \begin{cases}\left(x_{i j} x_{12} x_{13}\right) /\left(x_{1 i} x_{1 j} x_{23}\right) & \text { if } i, j \geq 4, \\ \left(x_{2 j} x_{13}\right) /\left(x_{1 j} x_{23}\right) & \text { if } i=2, \\ \left(x_{3 j} x_{12}\right) /\left(x_{1 j} x_{23}\right) & \text { if } i=3 .\end{cases}
$$

The proof breaks down into several cases, depending on how many of 1,2,3 lie in $\{i, j, k, l\}$. For example, if $1,2,3 \notin\{i, j, k, l\}$, then $z_{i j} z_{k l}-z_{i k} z_{j l}+z_{i l} z_{j k}=$ $\left(x_{i j} x_{k l}-x_{i k} x_{j l}+x_{i l} x_{j k}\right)\left(x_{12} x_{13}\right)^{2} /\left(x_{1 i} x_{1 j} x_{1 k} x_{1 l} x_{23}^{2}\right)$. The other cases to check are:

- $i=1,2,3 \notin\{j, k, l\}$,

- $i=1, j=2,3 \notin\{k, l\}$, or $j=3,2 \notin\{k, l\}$,

- $i=1, j=2, k=3$,

- $i=2,1,3 \notin\{j, k, l\}$ or $j=3,1,2 \notin\{i, k, l\}$, and

- $i=2, j=3,1 \notin\{k, l\}$.

In every case we see that the polynomial $\phi\left(z_{i j} z_{k l}-z_{i k} z_{j l}+z_{i l} z_{j k}\right)$ is equal to $x_{i j} x_{k l}-x_{i k} x_{j l}+x_{i l} x_{j k}$ times a monomial in the $x_{m n}$. This shows that $\phi$ takes a generating set for $J^{\prime}$ to a generating set for $I_{2, n} \subset \mathbb{k}\left[x_{i j}^{ \pm 1}: 1 \leq i<j \leq n\right]$, and thus

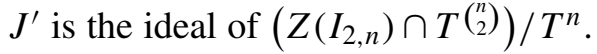

To see that $J=J^{\prime}$, it suffices to show that all other generators of $J^{\prime}$ lie in the ideal generated by these linear ones. Indeed,

$$
\begin{aligned}
& z_{i j} z_{k l}-z_{i k} z_{j l}+z_{i l} z_{j k} \\
& \quad=z_{i j}\left(z_{k l}-z_{2 l}+z_{2 k}\right)-z_{i k}\left(z_{j l}-z_{2 l}+z_{2 j}\right)+z_{i l}\left(z_{j k}-z_{2 k}+z_{2 j}\right) \\
& \quad+\left(z_{2 l}-z_{2 k}\right)\left(z_{i j}-z_{2 j}+z_{2 i}\right)+\left(z_{2 k}-z_{2 j}\right)\left(z_{i l}-z_{2 l}+z_{2 i}\right)+\left(z_{2 j}-z_{2 l}\right)\left(z_{i k}-z_{2 k}+z_{2 i}\right) .
\end{aligned}
$$

Remark 6.5. Consider the ideal $\tilde{J} \subset \mathbb{k}\left[z_{i j}: i j \in \mathscr{E} \cup\{23\}\right]$ obtained by homogenizing the ideal $J$ by adding the variable $z_{23}$. The variety $Z(\tilde{J}) \subseteq \mathbb{P}\left(\begin{array}{c}n-1 \\ 2\end{array}\right)-1$ is the linear subspace equal to the row space of the $(n-1) \times\left(\begin{array}{c}n-1 \\ 2\end{array}\right)$ matrix $\tilde{A}$ whose columns are the positive roots of the root system $A_{n-2}$. Specifically, the rows of $\tilde{A}$ are indexed by $2, \ldots, n$, and the columns are indexed by $\{\{i, j\}: 2 \leq i<j \leq n\}$, with $\tilde{A}_{i,\{j, k\}}$ equal to 1 if $i=j,-1$ if $i=k$, and zero otherwise. The variety $M_{0, n}$ is the intersection of $Z(\tilde{J}) \subseteq \mathbb{P}\left(\begin{array}{c}n-1 \\ 2\end{array}\right)-1$ with the torus $T^{\left(\begin{array}{c}n-1 \\ 2\end{array}\right)-1}$ of $\mathbb{P}^{\left(\begin{array}{c}n-1 \\ 2\end{array}\right)-1}$. This exhibits $M_{0, n}$ as a hyperplane complement and as a very affine variety in its intrinsic torus [Tevelev 2007].

Proof of Theorem 6.3. By Theorem $5.7 X_{\Delta}$ is a sufficiently large toric subvariety of $X_{\Sigma^{\star}}$, so Theorem 3.2 describes how to get equations for $\bar{M}_{0, n}$ inside $X_{\Delta}$. Equation (4) defines $V=\left(I_{\left(\begin{array}{c}n \\ 2\end{array}\right)} \mid C^{T}\right)$, so the map $v: \mathbb{k}\left[x_{i j}: 1 \leq i<j \leq n\right] \rightarrow \mathbb{k}\left[x_{I}^{ \pm 1}: I \in \mathscr{I}\right]$ 
of Theorem 3.2 is given by $v\left(x_{i j}\right)=x_{i j} \prod_{i, j \in I} x_{I}$, where the product is over $I \in \mathscr{I}$ with $1 \in I$, and $3 \leq|I| \leq n-3$. Thus, for $i, j, k$, and $l$ distinct, we have

$$
v\left(x_{i j} x_{k l}\right)=x_{i j} x_{k l} \prod_{i j \in I} x_{I} \prod_{k l \in I} x_{I},
$$

with the same restrictions on the products. Using the convention $x_{I}=x_{[n] \backslash I}$, we can write this as

$$
v\left(x_{i j} x_{k l}\right)=x_{i j} x_{k l} \prod_{\substack{i j \in I \\ k l \notin I}} x_{I} \prod_{\substack{1 \in i, j, k, l\} \cap I|=3\\| 1, i, j, k, l \in I}} x_{I} x_{I}^{2},
$$

where here the products are over all $I \in \mathscr{I}$ with $3 \leq|I| \leq n-3$, and there is no restriction that $1 \in I$ in the first product. Write

$$
M_{i j k l}=\prod_{\substack{1 \in I \\|\{i, j, k, l\} \cap I|=3}} x_{I} \prod_{1, i, j, k, l \in I} x_{I}^{2} .
$$

Then

$$
v\left(p_{i j k l}\right)=M_{i j k l}\left(\prod_{\substack{i j \in I \\ k l \notin I}} x_{I}-\prod_{\substack{i k \in I \\ j l \notin I}} x_{I}+\prod_{\substack{i l \in I \\ j k \notin I}} x_{I}\right) .
$$

Note that $v\left(p_{i j k l}\right)$ is already a polynomial, so there is no need to clear denominators. Thus by Theorem 3.2 the ideal $I_{\bar{M}_{0, n}}$ in the Cox ring of $X_{\Delta}$ is given by

$$
\begin{aligned}
I_{\bar{M}_{0, n}} & =\left(\left\langle\left(\prod_{\substack{i j \in I \\
l k \notin I}} x_{I}-\prod_{\substack{i k \in I \\
j l \notin I}} x_{I}+\prod_{\substack{i l \in I \\
j k \notin I}} x_{I}\right) M_{i j k l}\right\rangle:\left(\prod_{I \in \mathscr{I}} x_{I}\right)^{\infty}\right) \\
& =\left(\left\langle\prod_{\substack{i j \in I \\
k l \notin I}} x_{I}-\prod_{\substack{i k \in I \\
j l \notin I}} x_{I}+\prod_{\substack{i l \in I \\
j k \notin I}} x_{I}\right\rangle:\left(\prod_{I \in \mathscr{I}} x_{I}\right)^{\infty}\right),
\end{aligned}
$$

where the generating sets run over all $\{i, j, k, l\}$ with $1 \leq i<j<k<l \leq n$.

Example 6.6. (1) The case $n=5$ is covered in Examples 2.2, 3.5, and 4.5.

(2) When $n=6$, the ideal $I_{2,6}$ is generated by the equations $p_{i j k l}=x_{i j} x_{k l}-x_{i k} x_{j l}+$ $x_{i l} x_{j k}$, where $1 \leq i<j<k<l \leq 6$ in the ring $\mathbb{k}\left[x_{i j}: 1 \leq i<j \leq 6\right]$. The Cox ring of $X_{\Delta}$ is $\mathbb{k}\left[x_{I}: I \in \mathscr{I}\right]$. Applying the change of coordinates given by the matrix $R$ the $p_{i j k l}$ become $\tilde{p}_{i j k l}=x_{i j} x_{k l} x_{i j m} x_{i j n}-x_{i k} x_{j l} x_{i k m} x_{i k n}+x_{i l} x_{j k} x_{i l m} x_{i l n}$, where $\{i, j, k, l, m, n\}=\{1,2,3,4,5,6\}$. The ideal $I_{\bar{M}_{0,6}}$ has additional generators $q_{i j}=x_{i k} x_{j k} x_{i j k}^{2} x_{l m} x_{l n} x_{m n}-x_{i l} x_{j l} x_{i j l}^{2} x_{k m} x_{k n} x_{m n}$

$$
+x_{i m} x_{j m} x_{i j m}^{2} x_{k l} x_{k n} x_{l n}-x_{i n} x_{j n} x_{i j n}^{2} x_{k l} x_{k m} x_{l m},
$$

for $1 \leq i<j \leq 6$, where again $\{i, j, k, l, m, n\}=\{1, \ldots, 6\}$. 
Remark 6.7. When $n=4$ we can still follow the recipe of Section 3 to obtain the equations for $\bar{M}_{0,4} \cong \mathbb{P}^{1}$ inside $X_{\Sigma^{*}} \cong \mathbb{P}^{2}$. The Grassmannian $G(2,5) \subseteq \mathbb{P}^{5}$ is the hypersurface $Z\left(x_{12} x_{34}-x_{13} x_{24}+x_{14} x_{23}\right)$. In this case $M_{0,4}=Z\left(z_{34}-z_{24}+1\right)$ in $T^{2}=\operatorname{Spec}\left(\mathbb{k}\left[z_{24}^{ \pm 1}, z_{34}^{ \pm 1}\right]\right)$. Also

$$
R=\left(\begin{array}{llllll}
1 & 0 & -1 & -1 & 0 & 1 \\
0 & 1 & -1 & -1 & 1 & 0
\end{array}\right)\left(\begin{array}{lll}
1 & 0 & 0 \\
0 & 1 & 0 \\
0 & 0 & 1 \\
0 & 0 & 0 \\
0 & 0 & 0 \\
0 & 0 & 0 \\
0 & 0 & 0
\end{array}\right), \quad \text { so } V=\left(\begin{array}{llllll}
1 & 0 & 0 & 0 & 0 & 0 \\
0 & 1 & 0 & 0 & 0 & 0 \\
0 & 0 & 1 & 0 & 0 & 0
\end{array}\right) .
$$

Thus $v\left(x_{12} x_{34}-x_{13} x_{24}+x_{14} x_{23}\right)=y_{1}-y_{2}+y_{3} \subseteq \mathbb{k}\left[y_{1}, y_{2}, y_{3}\right]=\operatorname{Cox}\left(\mathbb{P}^{2}\right)$.

\section{VGIT and the effective cone of $\bar{M}_{0, n}$}

An important invariant of a projective variety $Y$ is its pseudoeffective cone $\overline{\operatorname{Eff}}(Y)$, and one of the primary goals of Mori theory is to understand the decomposition of this cone into Mori chambers. In this section we prove Theorem 7.1, which is the second part of Theorem 1.2. We identify a subcone $\mathscr{G}$ of the effective cone of $\bar{M}_{0, n}$ for which one has that $\bar{M}_{0, n}$ can be constructed as a GIT quotient of an affine variety with linearization determined by a given $D \in \mathscr{G}$. This prompts Question 7.4(3), which asks whether the cone $E$ of divisors spanned by boundary classes is a Mori dream region of the effective cone.

Let $H=\operatorname{Hom}\left(\mathrm{Cl}\left(X_{\Delta}\right), \mathbb{k}^{\times}\right) \cong\left(\mathbb{k}^{\times}\right)^{b+n}$. The torus $H$ acts on $\mathbb{A}^{|\mathscr{F}|}$ with weights given by the columns of the matrix $G$ of (5). Recall the cone

$$
\mathscr{G}\left(X_{\Delta}\right)=\bigcap_{\sigma \in \Delta} \operatorname{pos}\left(\left[D_{I}\right]: I \notin \sigma\right)
$$

from Definition 4.1. Let $i: \bar{M}_{0, n} \rightarrow X_{\Delta}$ be the inclusion of Theorem 5.7. The pullback $i^{*}\left(\mathscr{G}\left(X_{\Delta}\right)\right) \subset N^{1}\left(\bar{M}_{0, n}\right) \otimes \mathbb{R}$ is a subcone of the nef cone of $\bar{M}_{0, n}$.

Theorem 7.1. (1) For rational $\alpha \in \operatorname{int}\left(G\left(X_{\Delta}\right)\right)$ we have the GIT construction of $\bar{M}_{0, n}$ as

$$
\bar{M}_{0, n}=Z\left(I_{\bar{M}_{0, n}}\right) / / \alpha H,
$$

where $Z\left(I_{\bar{M}_{0, n}}\right) \subset \mathbb{A}^{|\mathscr{}|}$ is the affine subscheme defined by $I_{\bar{M}_{0, n}}$.

(2) Let $n \geq 5$. Given $\beta \in \mathbb{N} A_{n}$ there is $\alpha \in \mathbb{N} G$ for which

$$
Z\left(I_{\bar{M}_{0, n}}\right) / / \alpha=G(2, n) / / \beta T^{n-1},
$$

so all GIT quotients of $G(2, n)$ by $T^{n-1}$ can be obtained from $\bar{M}_{0, n}$ by variation of the GIT. 
Proof. The first part of the theorem is a direct application of Proposition 4.3. For the second, note that for $n \geq 5$ there are no repeated columns in the matrix $D$, so the result follows from Theorem 4.6 and Corollary 4.7.

Remark 7.2. (1) The second part of the theorem is still true for $n=4$, as $\bar{M}_{0,4} \cong$ $\mathbb{P}^{1}$, which is also equal to one of the GIT quotients of $G(2,4)$.

(2) When $n=5, I_{\bar{M}_{0,5}}=I_{2,5}$, so we see that $\bar{M}_{0,5}=G(2,5) / / \alpha T^{4}$ for $\alpha \in$ $\operatorname{int}\left(G\left(X_{\Delta}\right)\right)$. In this case the second part of the theorem is a tautology.

(3) For larger $n$, since $A$ and $C$ are nonnegative matrices, expression

$$
-\min \left\{(C u)_{i}: A u=\beta, u \in \mathbb{Q}_{\geq 0}^{m+1}\right\}
$$

of Theorem 4.6 is a nonpositive integer, and thus we can choose $\alpha=(\beta, 0)$.

Remark 7.3. A natural problem is to give a combinatorial description of these equations for $\bar{M}_{0, n}$, similar to that given for the GIT quotient $G(2, n) / / \beta T^{n-1}$ in [Howard et al. 2009]. Generators for the corresponding ring can still be described by graphs on $n$ vertices, but an added complication is that their Kempe lemma is not true; the generators corresponding to noncrossing graphs no longer give a basis for the degree-one part of the ring.

$\mathrm{Hu}$ and Keel Hu and Keel 2000 Mori dream spaces are introduced, which are varieties whose effective cones are polyhedral and for which the Mori chamber decomposition breaks this cone into a finite number of polyhedral pieces. They prove that if a variety $Y$ is a Mori dream space, then there is an embedding of $Y$ into a projective toric variety $X_{\Sigma}$, and thus a GIT construction of $Y$, so that $Y$ and $X_{\Sigma}$ have isomorphic Picard groups and effective cones, and all small $\mathbb{Q}$-factorial modifications of $Y$ can be obtained by variation of the GIT quotient from $Y$. They also define the weaker notion of subcone $C \subseteq \overline{\operatorname{Eff}}(Y)$ being a Mori dream region. This holds if

$$
R=\bigoplus_{D \in C} H^{0}(V, D)
$$

is finitely generated.

$\mathrm{Hu}$ and Keel raised the question of whether $\bar{M}_{0, n}$ is a Mori dream space. Theorem 5.7 shows that $\bar{M}_{0, n}$ embeds into $X_{\Delta}$ and Lemma 6.2 shows that $\operatorname{Pic}\left(\bar{M}_{0, n}\right) \cong$ $\mathrm{Cl}\left(X_{\Delta}\right)$. However, for $n \geq 6$, Keel and Vermeire showed that the cone $E$ generated by the boundary divisors of $\bar{M}_{0, n}$ is a proper subcone of $\operatorname{Eff}\left(\bar{M}_{0, n}\right)$ [Vermeire 2002], so the effective cones of $\bar{M}_{0, n}$ and $X_{\Delta}$ differ. On the other hand, Castravet [2009] showed $\bar{M}_{0,6}$ is indeed a Mori dream space. We believe that $E$ may be a Mori dream region. Indeed, the GIT chambers of $Z\left(I_{\bar{M}_{0, n}}\right) / / \alpha H$ divide $E$ into polyhedral chambers, each of which corresponds to a different compactification of $M_{0, n}$. The chamber containing $\mathscr{G}\left(X_{\Delta}\right)$ corresponds to the compactification $\bar{M}_{0, n}$. 
Question 7.4. (1) Let $\bar{M}_{0,6} \rightarrow X_{\Sigma}$ be the embedding of $\bar{M}_{0,6}$ into a toric variety of dimension 24 with isomorphic Picard group and effective cone guaranteed by the Mori dream space construction. Let $X_{\Sigma^{\prime}}$ be the toric subvariety obtained from $X_{\Sigma}$ by removing $T^{24}$-orbits of $X_{\Sigma}$ not intersecting $\bar{M}_{0,6}$. Is $X_{\Sigma^{\prime}}$ obtained from $X_{\Delta}$ in a natural way (such as by tropical modifications) that generalizes to $n>6$ ?

(2) Is there a toric embedding $\bar{M}_{0, n} \rightarrow X_{\Sigma}$ with $\bar{M}_{0, n}$ and $X_{\Sigma}$ having isomorphic Picard groups and effective cones that can be obtained from $X_{\Delta}$ by tropical modifications? This would support the conjecture that $\bar{M}_{0, n}$ is a Mori dream space.

(3) Let $E$ be the closed subcone of $\operatorname{Eff}\left(\bar{M}_{0, n}\right)$ spanned by the boundary divisors, let $S$ be the Cox ring of $X_{\Delta}$ and $I_{\bar{M}_{0, n}}$ the ideal of $\bar{M}_{0, n}$ in $S$. Is

$$
\left(S / I_{\bar{M}_{0, n}}\right)_{D} \cong H^{0}\left(\bar{M}_{0, n}, D\right)
$$

for all $D \in E$ ? This would imply that $E$ is a Mori dream region for $\bar{M}_{0, n}$.

Acknowledgements. We thank Klaus Altmann for helpful discussions about Chow quotients. We also thank Sean Keel and Jenia Tevelev for useful conversations. The authors were partially supported by NSF grants DMS-0509319 (Gibney) and DMS-0500386 (Maclagan).

\section{References}

[Bayer and Morrison 1988] D. Bayer and I. Morrison, "Standard bases and geometric invariant theory, I: Initial ideals and state polytopes”, J. Symbolic Comput. 6 (1988), 209-217. MR 90e:13001 Zbl 0675.13014

[Białynicki-Birula and Sommese 1987] A. Białynicki-Birula and A. J. Sommese, "A conjecture about compact quotients by tori”, pp. 59-68 in Complex analytic singularities, edited by T. Suwa and P. Wagreich, Adv. Stud. Pure Math. 8, North-Holland, Amsterdam, 1987. MR 88j:32042 Zbl 0616.14037

[Billera et al. 1990] L. J. Billera, P. Filliman, and B. Sturmfels, "Constructions and complexity of secondary polytopes”, Adv. Math. 83:2 (1990), 155-179. MR 92d:52028 Zbl 0714.52004

[Billera et al. 2001] L. J. Billera, S. P. Holmes, and K. Vogtmann, "Geometry of the space of phylogenetic trees”, Adv. in Appl. Math. 27:4 (2001), 733-767. MR 2002k:05229 Zbl 0995.92035

[Buneman 1974] P. Buneman, "A note on the metric properties of trees", J. Combinatorial Theory Ser. B 17 (1974), 48-50. MR 51 \#218 Zbl 0286.05102

[Castravet 2009] A.-M. Castravet, "The Cox ring of $\bar{M}_{0,6}$ ", Trans. Amer. Math. Soc. 361:7 (2009), 3851-3878. MR 2009m:14037 Zbl 1172.14010

[Cox 1995] D. A. Cox, "The homogeneous coordinate ring of a toric variety", J. Algebraic Geom. 4:1 (1995), 17-50. MR 95i:14046 Zbl 0846.14032

[Draisma 2008] J. Draisma, “A tropical approach to secant dimensions", J. Pure Appl. Algebra 212:2 (2008), 349-363. MR 2008j:14102 Zbl 1126.14059 
[Einsiedler et al. 2006] M. Einsiedler, M. Kapranov, and D. Lind, "Non-Archimedean amoebas and tropical varieties", J. Reine Angew. Math. 601 (2006), 139-157. MR 2007k:14038 Zbl 1115.14051

[Eisenbud 1995] D. Eisenbud, Commutative algebra, Graduate Texts in Mathematics 150, Springer, New York, 1995. MR 97a:13001 Zbl 0819.13001

[Feichtner 2006] E. M. Feichtner, "Complexes of trees and nested set complexes", Pacific J. Math. 227:2 (2006), 271-286. MR 2008a:05275 Zbl 1177.05031

[Feichtner and Yuzvinsky 2004] E. M. Feichtner and S. Yuzvinsky, "Chow rings of toric varieties defined by atomic lattices”, Invent. Math. 155:3 (2004), 515-536. MR 2004k:14009 Zbl 1083.14059

[Fulton 1993] W. Fulton, Introduction to toric varieties, Ann. Math. Studies 131, Princeton University Press, 1993. MR 94g:14028 Zbl 0813.14039

[Gathmann 2006] A. Gathmann, "Tropical algebraic geometry", Jahresber. Deutsch. Math.-Verein. 108:1 (2006), 3-32. MR 2007e:14088 Zbl 1109.14038

[Gel'fand et al. 1994] I. M. Gel'fand, M. M. Kapranov, and A. V. Zelevinsky, Discriminants, resultants, and multidimensional determinants, Birkhäuser, Boston, 1994. MR 1264417 (95e:14045) Zbl 0827.14036

[Howard et al. 2009] B. Howard, J. Millson, A. Snowden, and R. Vakil, "The equations for the moduli space of $n$ points on the line", Duke Math. J. 146:2 (2009), 175-226. MR 2009m:14070 Zbl 1161.14033

[Hu 2005] Y. Hu, “Topological aspects of Chow quotients”, J. Differential Geom. 69:3 (2005), 399_ 440. MR 2006f:14054 Zbl 1087.14032

[Hu and Keel 2000] Y. Hu and S. Keel, "Mori dream spaces and GIT”, Michigan Math. J. 48 (2000), 331-348. MR 2001i:14059 Zbl 1077.14554

[Jensen et al. 2008] A. N. Jensen, H. Markwig, and T. Markwig, "An algorithm for lifting points in a tropical variety”, Collect. Math. 59:2 (2008), 129-165. MR 2009a:14077 Zbl 1151.13021

[Kapranov 1993] M. M. Kapranov, "Chow quotients of Grassmannians, I", pp. 29-110 in I. M. Gel'fand Seminar, Adv. Soviet Math. 16, Amer. Math. Soc., Providence, RI, 1993. MR 95g:14053 Zbl 0811.14043

[Kapranov et al. 1992] M. M. Kapranov, B. Sturmfels, and A. V. Zelevinsky, "Chow polytopes and general resultants”, Duke Math. J. 67:1 (1992), 189-218. MR 93e:14062 Zbl 0780.14027

[Keel 1992] S. Keel, "Intersection theory of moduli space of stable $n$-pointed curves of genus zero", Trans. Amer. Math. Soc. 330:2 (1992), 545-574. MR 92f:14003 Zbl 0768.14002

[Keel and Tevelev 2006] S. Keel and J. Tevelev, "Geometry of Chow quotients of Grassmannians", Duke Math. J. 134:2 (2006), 259-311. MR 2007m:14017 Zbl 1107.14026

[Keel and Tevelev 2009] S. Keel and J. Tevelev, "Equations for $\bar{M}_{0, n}$ ", Internat. J. Math. 20:9 (2009), 1159-1184. MR 2011a:14059 Zbl 1187.14031

[Kollár 1996] J. Kollár, Rational curves on algebraic varieties, Ergebnisse der Mathematik (3) 32, Springer, Berlin, 1996. MR 98c:14001 Zbl 0877.14012

[Maclagan and Thomas 2007] D. Maclagan and R. R. Thomas, "Computational algebra and combinatorics of toric ideals", pp. 1-106 in Commutative algebra and combinatorics (Allahabad, 2003), vol. 1, edited by R. V. Gurjar et al., Ramanujan Math. Soc. Lect. Notes Ser. 4, Ramanujan Math. Soc., Mysore, 2007. MR 2009c:13069

[Mikhalkin 2006] G. Mikhalkin, "Tropical geometry and its applications", pp. 827-852 in International Congress of Mathematicians, vol. II, edited by J. L. V. Marta Sanz-Solé, Javier Soria and J. Verdera, Eur. Math. Soc., Zürich, 2006. MR 2008c:14077 Zbl 1103.14034 
[Mora and Robbiano 1988] T. Mora and L. Robbiano, "The Gröbner fan of an ideal”, J. Symbolic Comput. 6:2-3 (1988), 183-208. MR 90d:13004 Zbl 0668.13017

[Mustaţă 2002] M. Mustaţă, "Vanishing theorems on toric varieties", Tohoku Math. J. (2) 54:3 (2002), 451-470. MR 2003e:14013 Zbl 1092.14064

[Payne 2009] S. Payne, "Fibers of tropicalization", Math. Z. 262 (2009), 301-311. MR 2010c:14081 Zbl 05565288

[Robinson and Whitehouse 1996] A. Robinson and S. Whitehouse, "The tree representation of $\Sigma_{n+1}$ ", J. Pure Appl. Algebra 111:1-3 (1996), 245-253. MR 97g:55010 Zbl 0865.55010

[Speyer 2005] D. E. Speyer, Tropical geometry, Ph.D. thesis, University of California, Berkeley, 2005, Available at http://tinyurl.com/29947uz. MR 2707751

[Speyer and Sturmfels 2004] D. Speyer and B. Sturmfels, "The tropical Grassmannian", Adv. Geom. 4:3 (2004), 389-411. MR 2005d:14089 Zbl 1065.14071

[Sturmfels 1996] B. Sturmfels, Gröbner bases and convex polytopes, University Lecture Series 8 , American Mathematical Society, Providence, RI, 1996. MR 97b:13034

[Tevelev 2007] J. Tevelev, "Compactifications of subvarieties of tori”, Amer. J. Math. 129:4 (2007), 1087-1104. MR 2008f:14068 Zbl 1154.14039

[Vermeire 2002] P. Vermeire, “A counterexample to Fulton's conjecture on $\bar{M}_{0, n}$ ”, J. Algebra 248:2 (2002), 780-784. MR 2002k:14043 Zbl 1039.14014

[Vogtmann 1990] K. Vogtmann, "Local structure of some Out $\left(F_{n}\right)$-complexes", Proc. Edinburgh Math. Soc. (2) 33:3 (1990), 367-379. MR 92d:57002 Zbl 0694.20021

[Ziegler 1995] G. M. Ziegler, Lectures on polytopes, Graduate Texts in Mathematics 152, Springer, New York, 1995. MR 96a:52011 Zbl 0823.52002

Communicated by Ravi Vakil

Received 2009-05-29 Revised 2010-02-17 Accepted 2010-05-05

agibney@math.uga.edu

Department of Mathematics, University of Georgia, Athens, GA 30602, United States

D.Maclagan@warwick.ac.uk Mathematics Institute, Zeeman Building, University of Warwick, Coventry, CV4 7AL, United Kingdom 


\section{Algebra \& Number Theory}

www.jant.org

\section{EDITORS}

\section{MANAGING EDITOR}

Bjorn Poonen

Massachusetts Institute of Technology

Cambridge, USA

\author{
EDITORIAL BOARD CHAIR \\ David Eisenbud \\ University of California \\ Berkeley, USA
}

\section{BOARD OF EDITORS}

\section{Georgia Benkart}

Dave Benson

Richard E. Borcherds

John H. Coates

J-L. Colliot-Thélène

Brian D. Conrad

Hélène Esnault

Hubert Flenner

Edward Frenkel

Andrew Granville

Joseph Gubeladze

Ehud Hrushovski

Craig Huneke

Mikhail Kapranov

Yujiro Kawamata

János Kollár

Hendrik W. Lenstra

Yuri Manin

Barry Mazur
University of Wisconsin, Madison, USA

University of Aberdeen, Scotland

University of California, Berkeley, USA

University of Cambridge, UK

CNRS, Université Paris-Sud, France

University of Michigan, USA

Universität Duisburg-Essen, Germany

Ruhr-Universität, Germany

University of California, Berkeley, USA

Université de Montréal, Canada

San Francisco State University, USA

Hebrew University, Israel

University of Kansas, USA

Yale University, USA

University of Tokyo, Japan

Princeton University, USA

Universiteit Leiden, The Netherlands

Northwestern University, USA

Harvard University, USA
Susan Montgomery

Shigefumi Mori

Andrei Okounkov

Raman Parimala

Victor Reiner

Karl Rubin

Peter Sarnak

Michael Singer

Ronald Solomon

Vasudevan Srinivas

J. Toby Stafford

Bernd Sturmfels

Richard Taylor

Ravi Vakil

Michel van den Bergh

Marie-France Vignéras

Kei-Ichi Watanabe

Andrei Zelevinsky

Efim Zelmanov
University of Southern California, USA

RIMS, Kyoto University, Japan

Princeton University, USA

Emory University, USA

University of Minnesota, USA

University of California, Irvine, USA

Princeton University, USA

North Carolina State University, USA

Ohio State University, USA

Tata Inst. of Fund. Research, India

University of Michigan, USA

University of California, Berkeley, USA

Harvard University, USA

Stanford University, USA

Hasselt University, Belgium

Université Paris VII, France

Nihon University, Japan

Northeastern University, USA

University of California, San Diego, USA

\section{PRODUCTION}

ant@mathscipub.org

Silvio Levy, Scientific Editor

Andrew Levy, Production Editor

See inside back cover or www.jant.org for submission instructions.

The subscription price for 2010 is US \$140/year for the electronic version, and \$200/year (+\$30 shipping outside the US) for print and electronic. Subscriptions, requests for back issues from the last three years and changes of subscribers address should be sent to Mathematical Sciences Publishers, Department of Mathematics, University of California, Berkeley, CA 94720-3840, USA.

Algebra \& Number Theory (ISSN 1937-0652) at Mathematical Sciences Publishers, Department of Mathematics, University of California, Berkeley, CA 94720-3840 is published continuously online. Periodical rate postage paid at Berkeley, CA 94704, and additional mailing offices.

ANT peer review and production are managed by EditFLOW ${ }^{\mathrm{TM}}$ from Mathematical Sciences Publishers.

\section{PUBLISHED BY}

mathematical sciences publishers

http://www.mathscipub.org

A NON-PROFIT CORPORATION

Typeset in LATEX

Copyright $\odot 2010$ by Mathematical Sciences Publishers 


\section{Algebra \& Number Theory}

Volume $4 \quad$ No. $7 \quad 2010$

Hochschild cohomology and homology of quantum complete intersections

STEFFEN OPPERMANN

Meromorphic continuation for the zeta function of a Dwork hypersurface

THOMAS BARNET-LAMB

Equations for Chow and Hilbert quotients

ANGEla Gibney and Diane MaClagan

Haglund-Haiman-Loehr type formulas for Hall-Littlewood polynomials of type $B$ and C

\section{CRISTIAN LENART}

On exponentials of exponential generating series

ROLAND BACHER

On families of $\varphi, \Gamma$-modules

KIRAN KEDLAYA and RUOCHUAN LIU 